\title{
Synthesis and Reactivity of Azapalladacyclobutanes
}

\author{
Joshua E. Ney and John P. Wolfe*
}

Department of Chemistry, University of Michigan, 930 N. University Ave., Ann Arbor, MI 48209-1055

\section{Supporting Information}

Experimental procedures, characterization data for all new compounds, descriptions of deuterium labeling experiments, descriptions of structural and stereochemical assignments, and selected one- and two-dimensional NMR spectra (34 pages).

\section{Table of Contents}

General Considerations

Preparation and Characterization of Aziridine Substrates

Preparation and Characterization of Azapalladcyclobutanes

Alkene Insertion Reactions

Oxidative Cleavage Reactions

Assignment of Structure and Stereochemistry

Deuterium Labeling Experiment

Selected NMR Spectra

References
Page S2

Page S2

Page S8

Page S9

Page S10

Page S12

Page S13

Page S16

Page S34 


\section{General Considerations}

All reactions were carried out under a nitrogen atmosphere in flame-dried glassware. All reagents were obtained from commercial sources and were used without further purification. PhINTs, ${ }^{1}$ 2-Butyl-1-(toluene-4-sulfonyl)aziridine (5), ${ }^{2}$ 2-Methyl-1-(toluene-4-sulfonyl)aziridine $(\mathbf{9}),{ }^{3}$ 2-methyl-1-(nitrobenzene-4-sulfonyl)aziridine $(\mathbf{1 0}),{ }^{4}$ (bpy)Ni(cod) ${ }^{5}$ and $(E, E)-1,6-d^{2}-1,5-$ hexadiene $(\mathbf{2 1})^{6}$ were prepared according to literature procedures. The Pd-mediated conversion of 5 to $\mathbf{6}$ was described in a previous communication. ${ }^{7}$ Toluene, tetrahydrofuran, diethyl ether, and methylene chloride were purified using a GlassContour solvent purification system. Yields refer to isolated yields of compounds estimated to be $\geq 95 \%$ pure as determined by ${ }^{1} \mathrm{H}$ NMR analysis. The yields reported in the supporting information describe the result of a single experiment, whereas the yields reported in Tables 1-2, eq 3-4, and Schemes 2-3 are average yields of two or more experiments. Thus, the yields reported in the experimental section and supporting information may differ from those shown in Tables 1-2, eq 3-5, and Schemes 2-3.

\section{Preparation and Characterization of Aziridine Substrates}

2-But-3-enyl-1-(toluene-4-sulfonyl)aziridine (7). A flame-dried flask was cooled under a stream of nitrogen and charged with $\mathrm{Cu}(\mathrm{OTf})_{2}(320 \mathrm{mg}, 0.89 \mathrm{mmol})$. The flask was purged with nitrogen, and acetonitrile $(60 \mathrm{~mL})$ and 1,5-hexadiene $(6.3 \mathrm{~mL}, 4.34 \mathrm{~g}, 52.8 \mathrm{mmol})$ were added via syringe. Solid PhINTs $(6.57 \mathrm{~g}, 17.6 \mathrm{mmol})$ was added in a single portion, the flask was purged with nitrogen, and the mixture was stirred at room temperature until all PhINTs had dissolved (15 $\mathrm{min})$. The reaction mixture was then diluted with ethyl acetate $(60 \mathrm{~mL})$, filtered thru a pad of silica gel, and eluted with additional ethyl acetate $(120 \mathrm{~mL})$. The resulting solution was concentrated in vacuo, and the crude material was purified by flash chromatography on 
silica gel to afford the title compound $(2.88 \mathrm{~g}, 65 \%)$ as a colorless oil. ${ }^{1} \mathrm{H}$ NMR (500 MHz, $\left.\mathrm{CDCl}_{3}\right) \delta 7.80(\mathrm{~d}, J=8.0 \mathrm{~Hz}, 2 \mathrm{H}), 7.32(\mathrm{~d}, J=8.0 \mathrm{~Hz}, 2 \mathrm{H}), 5.75-5.66(\mathrm{~m}, 1 \mathrm{H}), 4.98-4.92(\mathrm{~m}$, $2 \mathrm{H}), 2.77-2.71(\mathrm{~m}, 1 \mathrm{H}), 2.60(\mathrm{~d}, J=7.0 \mathrm{~Hz}, 1 \mathrm{H}), 2.42(\mathrm{~s}, 3 \mathrm{H}), 2.06(\mathrm{~d}, J=4.5 \mathrm{~Hz}, 1 \mathrm{H}), 2.04-$ $1.95(\mathrm{~m}, 2 \mathrm{H}), 1.67-1.59(\mathrm{~m}, 1 \mathrm{H}), 1.47-1.38(\mathrm{~m}, 1 \mathrm{H}) ;{ }^{13} \mathrm{C} \mathrm{NMR}\left(125 \mathrm{MHz}, \mathrm{CDCl}_{3}\right) \delta 144.6$ 137.0, 135.1, 129.8, 128.1, 115.6, 39.9, 33.9, 30.9, 30.7, 21.8; IR (film) 1323, $1161 \mathrm{~cm}^{-1}$. Anal calcd for $\mathrm{C}_{13} \mathrm{H}_{17} \mathrm{NO}_{2} \mathrm{~S}$ : C, 62.12; H, 6.82; N, 5.57. Found: C, 62.34; H, 6.87; N, 5.54.

(E)-5-[1-(Toluene-4-sulfonyl)aziridin-2-yl]pent-2-enoic acid butyl ester (11). A flame-dried flask was cooled under a stream of nitrogen and charged with Grubb's $2^{\text {nd }}$ Generation Catalyst (133 mg, $0.16 \mathrm{mmol})$. The flask was purged with nitrogen, a solution of 7 (788 $\mathrm{mg}, 3.14 \mathrm{mmol}$ ) in methylene chloride $(15 \mathrm{~mL})$ was added via syringe followed by $n$-butyl acrylate $(899 \mu \mathrm{L}, 6.27$ $\mathrm{mmol}$ ), and the mixture was stirred at room temperature for $3 \mathrm{~h}$. The solvent was removed in vacuo and the residue was purified by flash chromatography on silica gel to afford the title compound (720 mg, $65 \%)$ as a brown oil. ${ }^{1} \mathrm{H}$ NMR $\left(500 \mathrm{MHz}, \mathrm{CDCl}_{3}\right) \delta 7.82(\mathrm{~d}, J=8.0 \mathrm{~Hz}, 2$ $\mathrm{H}), 7.35(\mathrm{~d}, J=8.0 \mathrm{~Hz}, 2 \mathrm{H}), 6.84(\mathrm{dt}, J=7.0,15.5 \mathrm{~Hz}, 1 \mathrm{H}), 5.74(\mathrm{dt}, J=1.5,15.5 \mathrm{~Hz}, 1 \mathrm{H})$, $4.12(\mathrm{t}, J=7.0 \mathrm{~Hz}, 2 \mathrm{H}), 2.77(\mathrm{~m}, 1 \mathrm{H}), 2.65(\mathrm{~d}, J=7.0 \mathrm{~Hz}, 1 \mathrm{H}), 2.45(\mathrm{~s}, 3 \mathrm{H}), 2.21-2.14(\mathrm{~m}, 2$ H), $2.09(\mathrm{~d}, J=5.0 \mathrm{~Hz}, 1 \mathrm{H}), 1.82-1.74(\mathrm{~m}, 1 \mathrm{H}), 1.67-1.60(\mathrm{~m}, 2 \mathrm{H}), 1.50-1.35(\mathrm{~m}, 3 \mathrm{H}), 0.94$ (t, 7.5 Hz, $3 \mathrm{H}) ;{ }^{13} \mathrm{C}$ NMR (125 MHz, $\left.\mathrm{CDCl}_{3}\right) \delta 166.7,147.0,144.9,135.1,130.0,128.3,122.5$, $64.5,39.5,34.1,30.9,30.0,29.5,21.9,19.4,14.0$; IR (film) 1717, 1324, $1161 \mathrm{~cm}^{-1}$. Anal calcd for $\mathrm{C}_{18} \mathrm{H}_{25} \mathrm{NO}_{4} \mathrm{~S}: \mathrm{C}, 61.51 ; \mathrm{H}, 7.17 ; \mathrm{N}, 3.99$. Found: C, 61.48; H, 7.35; N, 3.97.

(Z)-2-Pent-3-enyl-1-(toluene-4-sulfonyl)aziridine (12). A flame-dried flask was cooled under nitrogen and charged with 7 (2.15 g, $8.6 \mathrm{mmol})$ and methylene chloride $(86 \mathrm{~mL})$. The solution 
was cooled to $-78{ }^{\circ} \mathrm{C}$ and $\mathrm{O}_{3}$ was bubbled through the solution until a blue color persisted. A stream of nitrogen was bubbled through the solution until the blue color dissipated, triphenylphosphine $(2.69 \mathrm{~g}, 10.3 \mathrm{mmol})$ was added, and the reaction was allowed to warm to room temperature with stirring for $18 \mathrm{~h}$. The reaction mixture was concentraed in vacuo and the residue was purified by flash chromatography on silica gel to afford 3-[1-(toluene-4sulfonyl)aziridin-2-yl]propionaldehyde $(1.74 \mathrm{~g}, 80 \%)$ as a colorless oil. ${ }^{1} \mathrm{H}$ NMR (400 MHz, $\left.\mathrm{CDCl}_{3}\right) \delta 9.67(\mathrm{~s}, 1 \mathrm{H}), 7.81(\mathrm{~d}, J=8.4 \mathrm{~Hz}, 2 \mathrm{H}), 7.35(\mathrm{~d}, J=8.4 \mathrm{~Hz}, 2 \mathrm{H}), 2.84-2.76(\mathrm{~m}, 1 \mathrm{H})$, $2.64(\mathrm{~d}, J=7.2 \mathrm{~Hz}, 1 \mathrm{H}), 2.50-2.43(\mathrm{~m}, 2 \mathrm{H}), 2.46(\mathrm{~s}, 3 \mathrm{H}), 2.11(\mathrm{~d}, J=4.4 \mathrm{~Hz}, 1 \mathrm{H}), 2.11-2.02$ (m, $1 \mathrm{H}), 1.53-1.42(\mathrm{~m}, 1 \mathrm{H})$.

A flame-dried flask was cooled under a stream of nitrogen and charged with potassium tertbutoxide (830 mg, $7.4 \mathrm{mmol})$ and tetrahydrofuran $(40 \mathrm{~mL})$. Solid ethyltriphenylphosphonium bromide $(2.74 \mathrm{~g}, 7.4 \mathrm{mmol})$ was added in one portion, the flask was purged with nitrogen, and the mixture was stirred at room temperature for $30 \mathrm{~min}$. The reaction was then cooled to $-78{ }^{\circ} \mathrm{C}$, and a solution of 3-[1-(toluene-4-sulfonyl)aziridin-2-yl]propionaldehyde (1.7 g, $6.7 \mathrm{mmol})$ in tetrahydrofuran $(10 \mathrm{~mL})$ was added dropwise via syringe. The reaction was stirred at $-78{ }^{\circ} \mathrm{C}$ for $1.5 \mathrm{~h}$ and was then allowed to warm to room temperature and stir for $17 \mathrm{~h}$. The reaction was quenched with water $(40 \mathrm{~mL})$ and the resulting mixture extracted with ethyl acetate $(3 \mathrm{x} 40 \mathrm{~mL})$. The combined organic extracts were washed with brine $(40 \mathrm{~mL})$ and dried with anhydrous sodium sulfate. The solution was concentrated in vacuo, and the crude material was purified by flash chromatography on silica gel to afford the title compound $(1.2 \mathrm{~g}, 67 \%)$ as a colorless oil. ${ }^{1} \mathrm{H}$ NMR $\left(400 \mathrm{MHz}, \mathrm{CDCl}_{3}\right) \delta 7.81(\mathrm{~d}, J=8.0 \mathrm{~Hz}, 2 \mathrm{H}), 7.32(\mathrm{~d}, J=8.0 \mathrm{~Hz}, 2 \mathrm{H}), 5.46-5.37(\mathrm{~m}$, 1), 5.30-5.22 (m, 1 H), 2.76-2.69 (m, $1 \mathrm{H}), 2.62(\mathrm{~d}, J=7.2 \mathrm{~Hz}, 1 \mathrm{H}), 2.42(\mathrm{~s}, 3 \mathrm{H}), 2.07$ (d, $J=$ $4.8 \mathrm{~Hz}, 1 \mathrm{H}), 2.02-1.93(\mathrm{~m}, 2 \mathrm{H}), 1.62-1.54(\mathrm{~m}, 1 \mathrm{H}), 1.54-1.50$ (m, $3 \mathrm{H}), 1.45-1.35$ (m, $1 \mathrm{H})$; 
${ }^{13} \mathrm{C}$ NMR $\left(100 \mathrm{MHz}, \mathrm{CDCl}_{3}\right) \delta 144.6,135.3,129.8,128.7,128.1,125.2,40.2,33.9,31.4,24.3$, 21.8, 12.8; IR (film) 1323, $1161 \mathrm{~cm}^{-1}$. Anal calcd for $\mathrm{C}_{14} \mathrm{H}_{19} \mathrm{NO}_{2} \mathrm{~S}: \mathrm{C}, 63.36 ; \mathrm{H}, 7.22 ; \mathrm{N}, 5.28$. Found: C, 63.43; H, 7.25; N, 5.26.

(E/Z)-2-[4-(tert-Butyldimethylsilyloxy)but-3-enyl]-1-(toluene-4-sulfonyl)aziridine (13). A flame-dried flask was cooled under a stream of nitrogen and charged with $7(2.0 \mathrm{~g}, 8.0 \mathrm{mmol})$ and tetrahydrofuran $(8 \mathrm{~mL})$. A solution of 9-BBN $(19 \mathrm{~mL}, 0.5 \mathrm{M}$ in tetrahydrofuran, $9.5 \mathrm{mmol})$ was added dropwise and the resulting mixture was stirred at room temperature until $\mathbf{7}$ had been consumed as judged by capillary GC analysis $(4 \mathrm{~h})$. The reaction was cooled to $0{ }^{\circ} \mathrm{C}$, and $3.0 \mathrm{M}$ aqueous sodium hydroxide $(8 \mathrm{~mL})$ and $30 \%$ aqueous hydrogen peroxide $(8 \mathrm{~mL})$ were added sequentially. The mixture was allowed to warm to room temperature and was stirred for $2 \mathrm{~h}$. The reaction mixture was partitioned between ether $(40 \mathrm{~mL})$ and water $(40 \mathrm{~mL})$. The layers were separated, and the aqueous portion was extracted with ether $(2 \times 40 \mathrm{~mL})$. The combined organic extracts were washed with $10 \%$ aqueous sodium sulfite $(40 \mathrm{~mL})$ and brine $(40 \mathrm{~mL})$ and dried over anhydrous sodium sulfate. The solution was concentrated in vacuo and the residue was purified by flash chromatography on silica gel to afford 4-[1-(toluene-4-sulfonyl)aziridin-2yl]butan-1-ol (1.9 g, $89 \%)$ as a colorless oil. ${ }^{1} \mathrm{H}$ NMR (400 MHz, $\left.\mathrm{CDCl}_{3}\right) \delta 7.83(\mathrm{~d}, J=8.4 \mathrm{~Hz}$, $2 \mathrm{H}), 7.34(\mathrm{~d}, J=8.0 \mathrm{~Hz}, 2 \mathrm{H}), 3.60-3.54(\mathrm{~m}, 2 \mathrm{H}), 2.79-2.72(\mathrm{~m}, 1 \mathrm{H}), 2.62(\mathrm{~d}, J=7.2 \mathrm{~Hz}, 1$ H), 2.45 (s, $3 \mathrm{H}), 2.06(\mathrm{~d}, J=4.4 \mathrm{~Hz}, 1 \mathrm{H}), 1.69-1.59$ (m, $1 \mathrm{H}), 1.56-1.49$ (m, $2 \mathrm{H}), 1.42-1.32$ (m, $3 \mathrm{H}), 1.26-1.22(\mathrm{~m}, 1 \mathrm{H})$.

A flame-dried flask was cooled under a stream of nitrogen and charged with 4-[1-(toluene-4sulfonyl)aziridin-2-yl]butan-1-ol (1.9 $\mathrm{g}, \quad 7.1 \mathrm{mmol})$, methylene chloride $(60 \mathrm{~mL})$, and dimethylsulfoxide $(12 \mathrm{~mL})$. The mixture was cooled to $0{ }^{\circ} \mathrm{C}$ and triethylamine $(3.9 \mathrm{~mL}, 2.85 \mathrm{~g}$, 
$28.2 \mathrm{mmol})$ was added via syringe. Solid sulfur trioxide-pyridine complex (2.25 g, $14.1 \mathrm{mmol})$ was added and the resulting mixture was stirred at $0{ }^{\circ} \mathrm{C}$ for $3 \mathrm{~h}$. The reaction mixture was then diluted with ethyl acetate $(300 \mathrm{~mL})$ and washed with water $(400 \mathrm{~mL})$, saturated aqueous ammonium chloride $(200 \mathrm{~mL})$, and brine $(200 \mathrm{~mL})$. The organic layer was dried with anhydrous sodium sulfate and concentrated in vacuo. The residue was purified by flash chromatography on silica gel to afford 4-[1-(touene-4-sulfonyl)aziridin-2-yl]butyraldehyde $(1.4 \mathrm{~g}, 74 \%)$ as a colorless oil. ${ }^{1} \mathrm{H}$ NMR $\left(500 \mathrm{MHz}, \mathrm{CDCl}_{3}\right) \delta 9.69(\mathrm{t}, J=1.5 \mathrm{~Hz}, 1 \mathrm{H}), 7.83(\mathrm{~d}, J=8.0 \mathrm{~Hz}, 2 \mathrm{H})$, $7.35(\mathrm{~d}, J=8.0 \mathrm{~Hz}, 2 \mathrm{H}), 2.77-2.72(\mathrm{~m}, 1 \mathrm{H}), 2.63(\mathrm{~d}, J=7.0 \mathrm{~Hz}, 1 \mathrm{H}), 2.45(\mathrm{~s}, 3 \mathrm{H}), 2.43(\mathrm{dt}, J$ $=1.5,7.0 \mathrm{~Hz}, 2 \mathrm{H}), 2.06(\mathrm{~d}, J=4.5 \mathrm{~Hz}, 1 \mathrm{H}), 1.74-1.57(\mathrm{~m}, 3 \mathrm{H}), 1.37-1.30(\mathrm{~m}, 1 \mathrm{H})$.

A flame-dried flask was cooled under a stream of nitrogen and charged with potassium hydride (44 mg, $1.1 \mathrm{mmol})$ and tetrahydrofuran $(1 \mathrm{~mL})$, and the resulting suspension was cooled to $0{ }^{\circ} \mathrm{C}$. A solution of tert-butylchlorodimethylsilane $(151 \mathrm{mg}, 1.0 \mathrm{mmol})$ in tetrahydrofuran $(1 \mathrm{~mL})$ was added via syringe. A solution of 4-[1-(touene-4-sulfonyl)aziridin-2-yl]butyraldehyde (267 mg, $1.0 \mathrm{mmol})$ in tetrahydrofuran $(3 \mathrm{~mL})$ was added dropwise. The resulting mixture was allowed to warm to room temperature and was stirred at this temperature for $1 \mathrm{~h}$. At this time, saturated aqueous sodium bicarbonate $(20 \mathrm{~mL})$ and ethyl acetate $(20 \mathrm{~mL})$ were added, and the layers were separated. The organic phase was dried over anhydrous sodium sulfate and concentrated in vacuo. The residue was purified by flash chromatography on silica gel to afford the title compound (59 mg, $15 \%, E: Z=1: 1)$ as a colorless oil. Data are for the mixture. ${ }^{1} \mathrm{H}$ NMR (500 $\left.\mathrm{MHz}, \mathrm{CDCl}_{3}\right) \delta 7.82(\mathrm{~d}, J=8.5 \mathrm{~Hz}, 2 \mathrm{H}), 7.35-7.31(\mathrm{~m}, 2 \mathrm{H}), 6.21(\mathrm{dt}, J=1.5,12.0 \mathrm{~Hz}, 0.5 \mathrm{H})$, $6.14(\mathrm{dt}, J=1.5,5.5 \mathrm{~Hz}, 0.5 \mathrm{H}), 4.89(\mathrm{dt}, J=7.5,12.0 \mathrm{~Hz}, 0.5 \mathrm{H}), 4.36(\mathrm{dt}, J=5.5,7.0 \mathrm{~Hz}, 0.5$ H), 2.80-2.74 (m, $1 \mathrm{H}), 2.63(\mathrm{~d}, J=7.5 \mathrm{~Hz}, 0.5 \mathrm{H}), 2.60(\mathrm{~d}, J=7.0 \mathrm{~Hz}, 0.5 \mathrm{H}), 2.44(\mathrm{~s}, 1.5 \mathrm{H})$, 2.44 (s, $1.5 \mathrm{H}), 2.10-1.98$ (m, $2 \mathrm{H}), 1.96-1.83(\mathrm{~m}, 1 \mathrm{H}), 1.59-1.46$ (m, $1.5 \mathrm{H}), 1.43-1.35$ (m, 0.5 
H), $0.91(\mathrm{~s}, 4.5 \mathrm{H}), 0.90(\mathrm{~s}, 4.5 \mathrm{H}), 0.12(\mathrm{~s}, 3 \mathrm{H}), 0.10(\mathrm{~s}, 3 \mathrm{H}) ;{ }^{13} \mathrm{C} \mathrm{NMR}\left(125 \mathrm{MHz}, \mathrm{CDCl}_{3}\right) \delta$ 144.6, 144.5, 141.5, 139.6, 135.5, 135.4, 129.9, 129.8, 128.2, 128.2, 109.7, 108.5, 40.6, 40.0, 34. 2, 34.0, 32.6, 31.6, 25.9, 25.8, 25.0, 21.9, 21.2, 18.5, 18.4, -5.0, -5.2 (two aliphatic resonances are incidentally equivalent); IR (film) $1661,1325,1161 \mathrm{~cm}^{-1}$. Anal calcd for $\mathrm{C}_{19} \mathrm{H}_{31} \mathrm{NO}_{3} \mathrm{SSi}$ : C, 59.80; H, 8.19; N, 3.67. Found: C, 59.90; H, 8.27; N, 3.69.

2-Pent-4-enyl-1-(toluene-4-sulfonyl)aziridine (14). A flame-dried flask was charged with potassium tert-butoxide $(370 \mathrm{mg}, \quad 3.3 \mathrm{mmol})$ and tetrahydrofuran $(20 \mathrm{~mL})$. Solid methyltriphenylphosphonium bromide $(1.2 \mathrm{~g}, 3.3 \mathrm{mmol})$ was added in one portion, the flask was purged with nitrogen, and the mixture was stirred at room temperature for $30 \mathrm{~min}$. The reaction was then cooled to $-78{ }^{\circ} \mathrm{C}$, and a solution of 4-[1-(touene-4-sulfonyl)aziridin-2-yl]butyraldehyde ( $800 \mathrm{mg}, 3.0 \mathrm{mmol})$ in tetrahydrofuran $(10 \mathrm{~mL})$ was added dropwise via syringe. The reaction was stirred at $-78^{\circ} \mathrm{C}$ for $1 \mathrm{~h}$ and then was allowed to warm to room temperature and stir for $1 \mathrm{~h}$. The reaction was quenched with saturated aqueous ammonium chloride $(40 \mathrm{~mL})$. The reaction mixture was extracted with ethyl acetate $(3 \times 40 \mathrm{~mL})$, and the combined organic extracts were washed with brine $(40 \mathrm{~mL})$ and dried over anhydrous sodium sulfate. The solution was concentrated in vacuo, and the crude material was purified by flash chromatography on silica gel to afford the title compound $(720 \mathrm{mg}, 91 \%)$ as a colorless oil. ${ }^{1} \mathrm{H}$ NMR $\left(500 \mathrm{MHz}, \mathrm{CDCl}_{3}\right) \delta$ $7.82(\mathrm{~d}, J=8.0 \mathrm{~Hz}, 2 \mathrm{H}), 7.33(\mathrm{~d}, J=8.0 \mathrm{~Hz}, 2 \mathrm{H}), 5.73-5.64(\mathrm{~m}, 1 \mathrm{H}), 4.96-4.90(\mathrm{~m}, 2 \mathrm{H})$, 2.75-2.69 (m, $1 \mathrm{H}), 2.63(\mathrm{~d}, J=7.0 \mathrm{~Hz}, 1 \mathrm{H}), 2.44(\mathrm{~s}, 3 \mathrm{H}), 2.05(\mathrm{~d}, J=4.5 \mathrm{~Hz}, 1 \mathrm{H}), 2.02-1.96$ (m, $2 \mathrm{H}), 1.62-1.52(\mathrm{~m}, 1 \mathrm{H}), 1.38-1.28(\mathrm{~m}, 3 \mathrm{H}) ;{ }^{13} \mathrm{C} \mathrm{NMR}\left(125 \mathrm{MHz}, \mathrm{CDCl}_{3}\right) \delta$ 144.7, 138.2, 135.3, 129.8, 128.2, 115.1, 40.4, 34.0, 33.1, 30.8, 26.1, 21.8; IR (film) $1323,1160 \mathrm{~cm}^{-1}$. Anal calcd for $\mathrm{C}_{14} \mathrm{H}_{19} \mathrm{NO}_{2} \mathrm{~S}: \mathrm{C}, 63.36 ; \mathrm{H}, 7.22 ; \mathrm{N}, 5.28$. Found: C, 63.36; H, 7.29; N, 5.40. 


\section{Preparation and Characterization of Azapalladacyclobutane complexes}

(phen)Pd $\left.\mathbf{P N s C H}\left(\mathbf{C H}_{3}\right) \mathbf{C H}_{2}\right\}$ (15). Reaction of $363 \mathrm{mg}$ (1.5 mmol) of $\mathbf{1 0}$ with $458 \mathrm{mg}(0.5$ mmol) of $\mathrm{Pd}_{2}(\mathrm{dba})_{3}$ and $180 \mathrm{mg}(1.0 \mathrm{mmol})$ of 1,10-phenanthroline following the general procedure described in the experimental section of the article afforded $231 \mathrm{mg}(44 \%)$ of the title compound as an orange solid, m.p. $210{ }^{\circ} \mathrm{C}$ (decomp). ${ }^{1} \mathrm{H}$ NMR $\left(500 \mathrm{MHz}, \mathrm{CDCl}_{3}\right) \delta 10.00(\mathrm{dd}, J$ $=1.5,5.0 \mathrm{~Hz}, 1 \mathrm{H}), 8.55(\mathrm{dd}, J=1.5,5.0 \mathrm{~Hz}, 1 \mathrm{H}), 8.50(\mathrm{dd}, J=1.5,8.5 \mathrm{~Hz}, 1 \mathrm{H}), 8.47(\mathrm{dd}, J=$ 1.5, 8.0 Hz, $1 \mathrm{H}), 8.38(\mathrm{~d}, J=8.0 \mathrm{~Hz}, 2 \mathrm{H}), 8.19(\mathrm{~d}, J=9.0 \mathrm{~Hz}, 2 \mathrm{H}), 8.00-7.90(\mathrm{~m}, 3 \mathrm{H}), 7.73$ $(\mathrm{dd}, J=5.0,8.0 \mathrm{~Hz}, 1 \mathrm{H}), 4.65-4.58(\mathrm{~m}, 1 \mathrm{H}), 1.19(\mathrm{~d}, J=6.5 \mathrm{~Hz}, 3 \mathrm{H}), 1.19-1.15(\mathrm{~m}, 1 \mathrm{H})$, 0.62-0.59 (m, $1 \mathrm{H}) ;{ }^{13} \mathrm{C}$ NMR $\left(125 \mathrm{MHz}, \mathrm{CDCl}_{3}\right) \delta$ 153.0, 151.6, 149.8, 148.8, 146.9, 144.9, 137.6, 137.5, 130.1, 129.5, 128.9, 127.9, 126.6, 126.3, 125.0, 123.7, 65.2, 26.4, 3.0; IR (film) $1520,1346,1137,1085 \mathrm{~cm}^{-1}$.

(E)-(phen)Pd\{NTsCH[CH $\left.\left.\mathrm{CH}_{2} \mathrm{CH}_{2} \mathrm{CHCHC}(\mathrm{O}) \mathrm{OCH}_{2} \mathrm{CH}_{2} \mathrm{CH}_{2} \mathrm{CH}_{3}\right] \mathrm{CH}_{2}\right]$ (16). Reaction of 670 $\mathrm{mg}(1.9 \mathrm{mmol})$ of 11 with $582 \mathrm{mg}(0.64 \mathrm{mmol})$ of $\mathrm{Pd}_{2}(\mathrm{dba})_{3}$ and $229 \mathrm{mg}(1.3 \mathrm{mmol})$ of $1,10-$ phenanthroline in tetrahydrofuran $(130 \mathrm{~mL})$ following the general procedure afforded $360 \mathrm{mg}$ (44\%) of the title compound as an orange solid, m.p. $170{ }^{\circ} \mathrm{C}$ (decomp). ${ }^{1} \mathrm{H}$ NMR (500 MHz, $\left.\mathrm{CDCl}_{3}\right) \delta 9.95(\mathrm{dd}, J=1.5,4.5 \mathrm{~Hz}, 1 \mathrm{H}), 8.51(\mathrm{dd}, J=1.5,8.5 \mathrm{~Hz}, 1 \mathrm{H}), 8.43(\mathrm{dd}, J=1.5,5.0$ $\mathrm{Hz}, 1 \mathrm{H}), 8.38(\mathrm{dd}, J=1.5,8.5 \mathrm{~Hz}, 1 \mathrm{H}), 8.05(\mathrm{~d}, J=8.0 \mathrm{~Hz}, 2 \mathrm{H}), 7.92-7.87(\mathrm{~m}, 2 \mathrm{H}), 7.78(\mathrm{dd}$, $J=5.0,8.0 \mathrm{~Hz}, 1 \mathrm{H}), 7.66(\mathrm{dd}, J=5.5,8.0 \mathrm{~Hz}, 1 \mathrm{H}), 7.16(\mathrm{~d}, J=8.0 \mathrm{~Hz}, 2 \mathrm{H}), 6.88(\mathrm{dt}, J=7.0$ $15.5 \mathrm{~Hz}, 1 \mathrm{H}), 5.73(\mathrm{~d}, J=15.5 \mathrm{~Hz}, 1 \mathrm{H}), 4.52-4.44(\mathrm{~m}, 1 \mathrm{H}), 4.10(\mathrm{t}, J=7.0 \mathrm{~Hz}, 2 \mathrm{H}), 2.33(\mathrm{~s}, 3$ H), 2.19-2.13 (m, $2 \mathrm{H}), 1.66-1.47$ (m, $4 \mathrm{H}), 1.42-1.33(\mathrm{~m}, 2 \mathrm{H}), 1.06$ (dd, $J=5.0,8.5 \mathrm{~Hz}, 1 \mathrm{H})$, $0.92(\mathrm{t}, J=7.5 \mathrm{~Hz}, 3 \mathrm{H}), 0.49(\mathrm{dd}, J=5.0,5.0 \mathrm{~Hz}, 1 \mathrm{H}) ;{ }^{13} \mathrm{C} \mathrm{NMR}\left(125 \mathrm{MHz}, \mathrm{CDCl}_{3}\right) \delta 167.2$, $153.1,150.1,149.3,146.4,144.4,141.6,140.8,137.7,137.2,129.9,129.2,129.0,127.9,127.7$, 
126.7, 125.9, 124.9, 121.0, 68.3, 64.1, 37.5, 30.9, 27.7, 21.6, 19.4, 13.9, -6.5; IR (film) 1715, $1264,1132,1085 \mathrm{~cm}^{-1}$. MS (ESI) $\mathrm{m} / z 660.1146\left(660.1124\right.$ calcd for $\left.\mathrm{C}_{30} \mathrm{H}_{33} \mathrm{~N}_{3} \mathrm{O}_{4} \mathrm{PdS}, \mathrm{M}+\mathrm{Na}^{+}\right)$.

(bipy)Ni\{NTsCH( $\left.\left.\mathrm{CH}_{2} \mathrm{CH}_{2} \mathrm{CHCH}_{2}\right) \mathrm{CH}_{2}\right\}$ (8a). A flame-dried Schlenk flask was charged with (bpy)Ni(cod) (350 mg, $1.08 \mathrm{mmol})$ in a nitrogen-filled glovebox. A solution of 7 (299 mg, 1.19 mmol) in tetrahydrofuran $(70 \mathrm{~mL})$ was added, and the resulting mixture was stired at $65{ }^{\circ} \mathrm{C}$ for $5.5 \mathrm{~h}$. The reaction mixture was then cannula-filtered to a clean, flame-dried Schlenk flask under nitrogen. The tetrahydrofuran solution was concentrated under reduced pressure to a volume of 5 $\mathrm{mL}$. Hexane $(60 \mathrm{~mL})$ was added to afford a maroon precipitate. The solid material was collected by suction filtration in the glovebox, washed with hexane $(2 \times 20 \mathrm{~mL})$, and dried in vacuo to afford the title compound (406 mg, $81 \%$ ) as a maroon solid. ${ }^{1} \mathrm{H}$ NMR $\left(500 \mathrm{MHz}, \mathrm{CD}_{2} \mathrm{Cl}_{2}\right) \delta$ $9.43(\mathrm{~d}, J=5.0 \mathrm{~Hz}, 1 \mathrm{H}), 8.22(\mathrm{~d}, J=8.0 \mathrm{~Hz}, 2 \mathrm{H}), 7.98-7.87(\mathrm{~m}, 5 \mathrm{H}), 7.54-7.48(\mathrm{~m}, 1 \mathrm{H})$, 7.27-7.21 (m, $3 \mathrm{H}), 5.87-5.77(\mathrm{~m}, 1 \mathrm{H}), 5.01-4.85(\mathrm{~m}, 2 \mathrm{H}), 3.73-3.65(\mathrm{~m}, 1 \mathrm{H}), 2.38(\mathrm{~s}, 3 \mathrm{H})$, 2.10-2.02 (m, $2 \mathrm{H}), 1.74-1.66(\mathrm{~m}, 1 \mathrm{H}), 1.65-1.57(\mathrm{~m}, 1 \mathrm{H}), 0.29$ (dd, $J=5.0,8.5 \mathrm{~Hz}, 1 \mathrm{H}),-0$. $02(\mathrm{dd}, J=4.5,4.5 \mathrm{~Hz}, 1 \mathrm{H}) ;{ }^{13} \mathrm{C} \mathrm{NMR}\left(500 \mathrm{MHz}, \mathrm{CD}_{2} \mathrm{Cl}_{2}\right) \delta 155.8,152.0,151.8,149.6,143.1$, $140.5,139.8,138.3,137.5,128.8,127.6,126.6,126.1,121.6,120.4,113.5,64.6,39.1,29.8,21.3$ -5.9 .

\section{Alkene Insertion Reactions (phen) Pd $\left\{\mathrm{NTsCH}\left(\mathrm{CH}_{2} \mathrm{CH}_{2}\right) \mathrm{CH}_{2} \mathrm{CHCH}\left(\mathrm{C}(\mathrm{O}) \mathrm{OCH}_{2} \mathrm{CH}_{2} \mathrm{CH}_{2} \mathrm{CH}_{3}\right)\right\}$}

(18). Azapalladacyclobutane 16 (95 mg, $0.15 \mathrm{mmol}$ ) was converted to the title compound using $28 \mathrm{mg}$ of $\mathrm{CuI}(0.15 \mathrm{mmol})$ following the procedure described in the experimental section of the article for the conversion of $\mathbf{8}$ to $\mathbf{1 7}$ except tetrahydrofuran $(15 \mathrm{~mL})$ was used as the reaction solvent, 
the reaction was conducted at $65{ }^{\circ} \mathrm{C}$, and pentane was used to precipitate the product. This modified procedure afforded the title compound (56 mg, $59 \%$ ) as an orange solid, m.p. $200{ }^{\circ} \mathrm{C}$ (decomp). ${ }^{1} \mathrm{H}$ NMR (500 MHz, $\left.\mathrm{CDCl}_{3}\right) \delta 9.69(\mathrm{dd}, J=1.5,5.0 \mathrm{~Hz}, 1 \mathrm{H}), 9.55(\mathrm{~d}, J=5.0 \mathrm{~Hz}, 1$ H), $8.48(\mathrm{~d}, J=7.5 \mathrm{~Hz}, 1 \mathrm{H}), 8.40(\mathrm{dd}, J=1.5,8.5 \mathrm{~Hz}, 1 \mathrm{H}), 7.92(\mathrm{~s}, 2 \mathrm{H}), 7.90-7.86(\mathrm{~m}, 3 \mathrm{H})$, $7.77(\mathrm{dd}, J=5.0,8.0 \mathrm{~Hz}, 1 \mathrm{H}), 7.19(\mathrm{~d}, J=8.0 \mathrm{~Hz}, 2 \mathrm{H}), 3.98(\mathrm{dt}, J=7.0,11.0 \mathrm{~Hz}, 1 \mathrm{H}), 3.87$ $(\mathrm{dt}, J=7.0,11.0 \mathrm{~Hz}, 1 \mathrm{H}), 3.72(\mathrm{dd}, J=4.5,6.5 \mathrm{~Hz}, 1 \mathrm{H}), 3.19(\mathrm{~d}, J=1.5 \mathrm{~Hz}, 1 \mathrm{H}), 2.38(\mathrm{~s}, 3 \mathrm{H})$, 2.10-2.03 (m, $1 \mathrm{H}), 1.98-1.88(\mathrm{~m}, 2 \mathrm{H}), 1.83-1.76(\mathrm{~m}, 1 \mathrm{H}), 1.72-1.64(\mathrm{~m}, 2 \mathrm{H}), 1.52-1.44(\mathrm{~m}$, $2 \mathrm{H}), 1.36-1.23(\mathrm{~m}, 3 \mathrm{H}), 0.88(\mathrm{t}, J=7.5 \mathrm{~Hz}, 3 \mathrm{H}) ;{ }^{13} \mathrm{C} \mathrm{NMR}\left(125 \mathrm{MHz}, \mathrm{CDCl}_{3}\right)$ \& 178.2, 152.0, 151.6, 147.9, 145.1, 143.0, 140.0, 138.0, 137.2, 129.9, 129.4, 129.0, 128.0, 127.4, 126.6, 125.2, 124.4, 63.2, 55.1, 38.5, 37.6, 34.4, 31.0, 30.8, 30.1, 21.7, 19.7, 14.1; IR (film) 1694, 1426, 1263 , 1127, $1088 \mathrm{~cm}^{-1}$. MS (ESI) $\mathrm{m} / z 660.1134\left(660.1124\right.$ calcd for $\left.\mathrm{C}_{30} \mathrm{H}_{33} \mathrm{~N}_{3} \mathrm{O}_{4} \mathrm{PdS}, \mathrm{M}+\mathrm{Na}^{+}\right)$.

\section{Oxidative Cleavage Reactions}

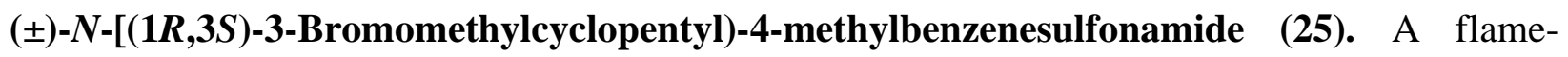
dried flask was charged with 17 (91 mg, $0.169 \mathrm{mmol})$ and copper (II) bromide (76 mg, 0.338 mmol). The flask was purged with nitrogen, and methylene chloride $(17 \mathrm{~mL})$ was added. The reaction was stirred for $14 \mathrm{~h}$ at room temperature, during which time a rust-colored precipitate formed. The mixture was treated with $1 \mathrm{M}$ aqueous hydrochloric acid $(20 \mathrm{~mL})$ and the layers were separated. The aqueous portion was extracted with methylene chloride $(3 \times 20 \mathrm{~mL})$. The combined organic extracts were washed with saturated aqueous sodium bicarbonate $(20 \mathrm{~mL})$ and brine $(20 \mathrm{~mL})$, dried with anhydrous sodium sulfate, and concentrated in vacuo. The crude material was purified by flash chromatography on silica gel to afford the title compound ( $31 \mathrm{mg}$, $55 \%)$ as a colorless oil. ${ }^{1} \mathrm{H}$ NMR $\left(500 \mathrm{MHz}, \mathrm{CDCl}_{3}\right) \delta 7.77(\mathrm{~d}, J=8.5 \mathrm{~Hz}, 2 \mathrm{H}), 7.32(\mathrm{~d}, J=8.0$ 
Hz, 2 H), 4.88 (d, J = 7.0 Hz, 1 H), 3.66-3.58 (m, 1 H), 3.39-3.32 (m, 2 H), 2.44 (s, 3 H), 2.31$2.20(\mathrm{~m}, 1 \mathrm{H}), 2.13-2.06(\mathrm{~m}, 1 \mathrm{H}), 1.87-1.72(\mathrm{~m}, 2 \mathrm{H}), 1.54-1.42(\mathrm{~m}, 2 \mathrm{H}), 1.23-1.16(\mathrm{~m}, 1 \mathrm{H})$; ${ }^{13} \mathrm{C}$ NMR $\left(100 \mathrm{MHz}, \mathrm{CDCl}_{3}\right) \delta 143.7,137.8,130.0,127.3,54.8,39.9,39.3,38.8,33.1,28.8$, 21.8; IR (film) 3270, 1322, 1158, $1093 \mathrm{~cm}^{-1}$. Anal calcd for $\mathrm{C}_{13} \mathrm{H}_{18} \mathrm{BrNO}_{2} \mathrm{~S}$ : C, 46.99; H, 5.46; N, 4.22. Found: C, 47.12; H, 5.59; N, 4.25.

Acetic acid (1R,3S)-3-(toluene-4-sulfonylamino)cyclopentylmethyl ester (26). A flame-dried flask was charged with 17 (53 mg, $0.1 \mathrm{mmol})$ and iodobenzene diacetate $(53 \mathrm{mg}, 0.1 \mathrm{mmol})$. The flask was purged with nitrogen, and methylene chloride $(10 \mathrm{~mL})$ was added. The reaction was stirred for $22 \mathrm{~h}$ at room temperature and was then treated with saturated aqueous ammonium chloride $(20 \mathrm{~mL})$ and the layers were separated. The aqueous portion was extracted with methylene chloride $(3 \times 20 \mathrm{~mL})$. The combined organic extracts were dried with anhydrous sodium sulfate and concentrated in vacuo. The crude material was purified by flash chromatography on silica gel to afford the title compound (10 $\mathrm{mg}, 32 \%)$ as a colorless oil. This material contained about $15 \%$ of an unidentified impurity. Data are for the major product. ${ }^{1} \mathrm{H}$ NMR (500 MHz, CDCl $) \delta 7.75(\mathrm{~d}, J=8.5 \mathrm{~Hz}, 2 \mathrm{H}), 7.30(\mathrm{~d}, J=8.0 \mathrm{~Hz}, 2 \mathrm{H}), 4.69$ (br d, $J=7.5$ $\mathrm{Hz}, 1 \mathrm{H}), 3.97-3.90(\mathrm{~m}, 2 \mathrm{H}), 3.64-3.56(\mathrm{~m}, 1 \mathrm{H}), 2.43$ (s, $3 \mathrm{H}), 2.22-2.12(\mathrm{~m}, 1 \mathrm{H}), 2.06-1.99$ (m, $1 \mathrm{H}), 2.04$ (s, $3 \mathrm{H}), 1.84-1.76(\mathrm{~m}, 1 \mathrm{H}), 1.72-1.64(\mathrm{~m}, 1 \mathrm{H}), 1.49-1.35(\mathrm{~m}, 2 \mathrm{H}), 1.15-1.07$ (m, $1 \mathrm{H})$.

In order to more firmly establish the identity of $\mathbf{2 6}$, the acetate group was cleaved through treatment of a solution of $\mathbf{2 6}(10 \mathrm{mg}, 0.032 \mathrm{mmol})$ in methanol $(1 \mathrm{~mL})$ with solid potassium carbonate $(10 \mathrm{mg}, 0.072 \mathrm{mmol})$. The resulting mixture was stirred at room temperature for $2 \mathrm{~h}$. Water $(10 \mathrm{~mL})$ was added, followed by ethyl acetate $(10 \mathrm{~mL})$. The layers were separated, and the 
organic portion was dried with anhydrous sodium sulfate and concentrated in vacuo. The crude material was purified by flash chromatography on silica gel to afford the title compound (4 mg, $46 \%)$ as a colorless oil. ${ }^{1} \mathrm{H}$ NMR $\left(500 \mathrm{MHz}, \mathrm{CDCl}_{3}\right) \delta 7.75(\mathrm{~d}, J=8.5 \mathrm{~Hz}, 2 \mathrm{H}), 7.29(\mathrm{~d}, J=8.5$ $\mathrm{Hz}, 2 \mathrm{H}), 5.15$ (br d, $J=7.5 \mathrm{~Hz}, 1 \mathrm{H}), 3.70-3.62(\mathrm{~m}, 1 \mathrm{H}), 3.55(\mathrm{~d}, J=5.0 \mathrm{~Hz}, 2 \mathrm{H}), 2.43(\mathrm{~s}, 3$ H), 2.18-2.08 (m, 1 H), 2.00-1.93 (m, 1 H), 1.74-1.64 (m, 2 H), 1.61 (br s, 1 H), 1.56-1.45 (m, $2 \mathrm{H}), 1.23-1.16(\mathrm{~m}, 1 \mathrm{H}) ;{ }^{13} \mathrm{C} \mathrm{NMR}\left(100 \mathrm{MHz}, \mathrm{CDCl}_{3}\right) \delta 143.3,138.4,129.9,127.3,66.1,55.0$, 39.0, 36.8, 33.9, 25.8, 21.8; IR (film) 3504, 3267, 1322, 1156, $1094 \mathrm{~cm}^{-1}$. MS (ESI) $\mathrm{m} / z$ 292.0977 (292.0983 calcd for $\mathrm{C}_{13} \mathrm{H}_{19} \mathrm{NO}_{3} \mathrm{~S}, \mathrm{M}+\mathrm{Na}^{+}$).

\section{Assignment of Structure and Stereochemistry}

\section{(phen)Pd $\left\{\mathrm{NTsCH}\left(\mathrm{CH}_{2} \mathrm{CH}_{2}\right) \mathrm{CH}_{2} \mathrm{CHCH}\left(\mathrm{C}(\mathrm{O}) \mathrm{OCH}_{2} \mathrm{CH}_{2} \mathrm{CH}_{2} \mathrm{CH}_{3}\right)\right\}$ (18)}

The C-bound enolate configuration of 19 was established by ${ }^{13} \mathrm{C}$ NMR analysis. The chemical shift of the carbonyl carbon in palladium enolate complexes correlates in a predictable manner with enolate configuration. ${ }^{8}$ Whereas C-bound enolate carbonyl carbon resonances appear in the region characteristic of organic carbonyls (170-210 ppm), those of the corresponding O-bound enolates generally appear much further upfield (130-150 ppm). The resonance attributable to the carbonyl carbon $\left(\mathrm{C}_{\mathrm{A}}\right)$ in $\mathbf{1 8}$ appears at a chemical shift of 178.2 ppm, characteristic of an ester carbonyl. Thus, $\mathbf{1}$ was assigned as a C-bound enolate.

The relative stereochemistry of $\mathbf{1 8}$ was assigned on the basis of the difference nOe signal shown below. 


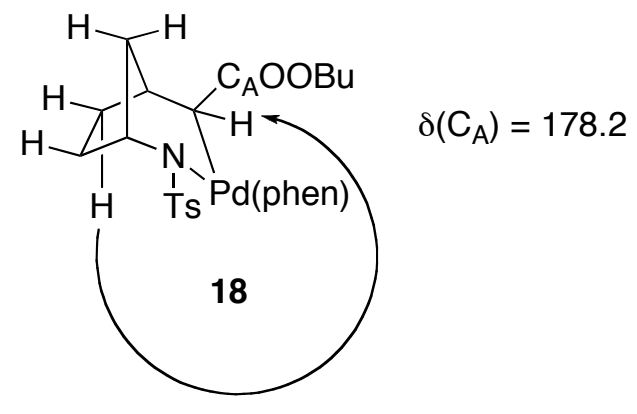

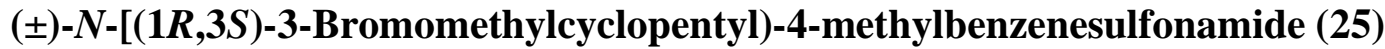

The relative stereochemistry of $\mathbf{2 5}$ was assigned on the basis of difference nOe signals as shown below. The relative stereochemistry of $\mathbf{2 6}$ was assigned based on analogy to this example.

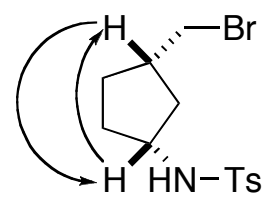

25

\section{Deuterium Labeling Experiment}

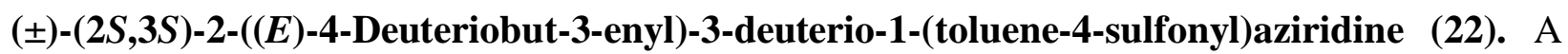
flame-dried flask was cooled under a stream of nitrogen and charged with $\mathrm{Cu}(\mathrm{OTf})_{2}(134 \mathrm{mg}$, $0.37 \mathrm{mmol})$. The flask was purged with nitrogen, and acetonitrile $(20 \mathrm{~mL})$ and $(E, E)-1,6-d^{2}-1,5-$ hexadiene (22, $1.86 \mathrm{~g}, 22.0 \mathrm{mmol})$ were added via syringe. Solid PhINTs (2.76 g, $7.4 \mathrm{mmol})$ was added in a single portion, the flask was purged with nitrogen, and the mixture was stirred at room temperature until all PhINTs had dissolved (15 min). The reaction mixture was diluted with ethyl acetate $(20 \mathrm{~mL})$, filtered thru a pad of silica gel, and eluted with additional ethyl acetate (120 $\mathrm{mL}$ ). The resulting solution was concentrated in vacuo, and the crude material was purified by flash chromatography on silica gel to afford the title compound (980 mg, $52 \%)$ as a colorless oil. 
This material was isolated as 5:1 mixture of trans:cis aziridines and a ca. 5:1 E:Z mixture of olefin isomers as judged by ${ }^{1} \mathrm{H}$ NMR analysis. Data are for the major isomer. ${ }^{1} \mathrm{H}$ NMR $(500$ $\left.\mathrm{MHz}, \mathrm{CDCl}_{3}\right) \delta 7.82(\mathrm{~d}, J=8.0 \mathrm{~Hz}, 2 \mathrm{H}), 7.34(\mathrm{~d}, J=8.0 \mathrm{~Hz}, 2 \mathrm{H}), 5.76-5.68(\mathrm{~m}, 1 \mathrm{H}), 4.95(\mathrm{dt}$, $J=1.5,17.0 \mathrm{~Hz}, 1 \mathrm{H}), 2.78-2.73(\mathrm{~m}, 1 \mathrm{H}), 2.44(\mathrm{~s}, 3 \mathrm{H}), 2.06(\mathrm{~d}, J=5.0 \mathrm{~Hz}, 1 \mathrm{H}), 2.09-1.97(\mathrm{~m}$, $2 \mathrm{H}), 1.68-1.61(\mathrm{~m}, 1 \mathrm{H}), 1.49-1.41(\mathrm{~m}, 1 \mathrm{H}) ;{ }^{2} \mathrm{H} \mathrm{NMR}\left(77 \mathrm{MHz}, \mathrm{CHCl}_{3}\right) \delta 5.00$ (s, $\left.1 \mathrm{D}\right), 2.64$ $(\mathrm{s}, 1 \mathrm{D}) ;{ }^{13} \mathrm{C}$ NMR $\left(125 \mathrm{MHz}, \mathrm{CDCl}_{3}\right) \delta 144.7,137.0,135.3,129.8,128.2,115.5\left(\mathrm{t}, J_{\mathrm{CD}}=24.0\right.$ $\mathrm{Hz}), 39.9,33.8\left(\mathrm{t}, J_{\mathrm{CD}}=25.9 \mathrm{~Hz}\right), 30.9,30.8,21.8$; IR (film) 1322, $1158 \mathrm{~cm}^{-1}$. MS (ESI) $\mathrm{m} / z$ 276.0994 (276.1003 calcd for $\mathrm{C}_{13} \mathrm{H}_{15} \mathrm{D}_{2} \mathrm{NO}_{2} \mathrm{~S}, \mathrm{M}+\mathrm{Na}^{+}$).

(phen)Pd\{NTsCH(CH2 $\mathbf{C H}_{2} \mathbf{C H C H D ) C H D \}}$ (23). Reaction of $253 \mathrm{mg}$ (1.0 mmol) of 23 with $458 \mathrm{mg}(0.5 \mathrm{mmol})$ of $\mathrm{Pd}_{2}(\mathrm{dba})_{3}$ and $180 \mathrm{mg}(1.0 \mathrm{mmol})$ of 1,10-phenanthroline following the general procedure described in the experimental section of the Article for the oxidative addition of aziridines to $\mathrm{Pd}_{2}(\mathrm{dba})_{3} /$ phen afforded $271 \mathrm{mg}(50 \%)$ of the title compound as an orange solid, m.p. $185^{\circ} \mathrm{C}$ (decomp). This material was isolated as 5:1 mixture of cis:trans metallacycles and a 5:1 E:Z mixture of olefin isomers as judged by ${ }^{1} \mathrm{H}$ NMR analysis. Data are for the major isomer. ${ }^{1} \mathrm{H}$ NMR $\left(500 \mathrm{MHz}, \mathrm{CDCl}_{3}\right) \delta 10.07(\mathrm{dd}, J=1.5,4.5 \mathrm{~Hz}, 1 \mathrm{H}), 8.55(\mathrm{dd}, J=1.5,5.5 \mathrm{~Hz}, 1 \mathrm{H})$, $8.49(\mathrm{dd}, J=1.5,8.0 \mathrm{~Hz}, 1 \mathrm{H}), 8.40(\mathrm{dd}, J=2.0,8.5 \mathrm{~Hz}, 1 \mathrm{H}), 8.09(\mathrm{~d}, J=8.0 \mathrm{~Hz}, 2 \mathrm{H}), 7.92(\mathrm{~s}$, $1 \mathrm{H}), 7.92(\mathrm{~s}, 1 \mathrm{H}), 7.84(\mathrm{dd}, J=5.0,8.0 \mathrm{~Hz}, 1 \mathrm{H}), 7.69(\mathrm{dd}, J=5.0,8.0 \mathrm{~Hz}, 1 \mathrm{H}), 7.16(\mathrm{~d}, J=$ $8.0 \mathrm{~Hz}, 2 \mathrm{H}), 5.74(\mathrm{dt}, J=6.5,17.0 \mathrm{~Hz}, 1 \mathrm{H}), 4.92(\mathrm{dt}, J=1.5,17.0 \mathrm{~Hz}, 1 \mathrm{H}), 4.50-4.45(\mathrm{~m}, 1$ $\mathrm{H}), 2.34$ (s, $3 \mathrm{H}), 2.07-1.94(\mathrm{~m}, 2 \mathrm{H}), 1.61-1.48(\mathrm{~m}, 2 \mathrm{H}), 1.09$ (d, J = 8.0 Hz, $1 \mathrm{H}) ;{ }^{2} \mathrm{H}$ NMR $(77$ $\left.\mathrm{MHz}, \mathrm{CHCl}_{3}\right) \delta 4.90$ (s, $\left.1 \mathrm{D}\right), 0.59$ (s, $\left.1 \mathrm{D}\right) ;{ }^{13} \mathrm{C} \mathrm{NMR}\left(125 \mathrm{MHz}, \mathrm{CDCl}_{3}\right) \delta 153.5,149.5,146.8$, $144.7,141.8,140.6,139.4,137.5,137.1,129.9,129.3,128.9,128.0,127.8,126.5,126.1,124.8$, $68.6,38.5,29.0,21.6$ (the resonances of the two carbon atoms bound to deuterium atoms were 
not visible in the one-dimensional ${ }^{13} \mathrm{C}$ NMR spectrum due to an insufficient signal-to-noise ratio; the chemical shifts of these carbon resonances were determined to be 113.4 and $-6.6 \mathrm{ppm}$ by HSQC analysis); IR (film) $1127,1085 \mathrm{~cm}^{-1}$. MS (ESI) $\mathrm{m} / z 562.0727$ (562.0725 calcd for $\left.\mathrm{C}_{25} \mathrm{H}_{23} \mathrm{D}_{2} \mathrm{~N}_{3} \mathrm{O}_{2} \mathrm{PdS}, \mathrm{M}+\mathrm{Na}^{+}\right)$.

(phen)Pd\{NTsCH( $\left.\left.\mathbf{C H}_{2} \mathbf{C H}_{2}\right) \mathbf{C H D C H C H D}\right\}$ (24). The reaction of $\mathbf{2 3}$ (135 mg, $0.25 \mathrm{mmol}$ ) and $\mathrm{CuI}(9.5 \mathrm{mg}, 0.05 \mathrm{mmol})$ in methylene chloride $(25 \mathrm{~mL})$ following the procedure described in the experimental section of the article for the conversion of $\mathbf{8}$ to $\mathbf{1 7}$ afforded the title compound (91 mg, $67 \%$ ) as a yellow-orange solid, m.p. $195{ }^{\circ} \mathrm{C}$ (decomp). This material was isolated as 5:1 mixture of $\mathrm{C} 3$ epimers and a 5:1 mixture of $\mathrm{C} 8$ epimers as judged by ${ }^{1} \mathrm{H}$ NMR analysis. Data are for the major isomer. ${ }^{1} \mathrm{H}$ NMR $\left(500 \mathrm{MHz}, \mathrm{CDCl}_{3}\right) \delta 9.65(\mathrm{~d}, J=4.5 \mathrm{~Hz}, 1 \mathrm{H}), 8.85(\mathrm{~d}, J=4.5$ $\mathrm{Hz}, 1 \mathrm{H}), 8.49(\mathrm{~d}, J=7.5 \mathrm{~Hz}, 1 \mathrm{H}), 8.36(\mathrm{~d}, J=8.0 \mathrm{~Hz}, 1 \mathrm{H}), 8.12(\mathrm{~d}, J=8.0 \mathrm{~Hz}, 2 \mathrm{H}), 7.91-7.84$ (m, $3 \mathrm{H}), 7.77(\mathrm{dd}, J=5.0,7.5 \mathrm{~Hz}, 1 \mathrm{H}), 7.21(\mathrm{~d}, J=8.0 \mathrm{~Hz}, 2 \mathrm{H}), 3.84(\mathrm{~d}, J=6.0 \mathrm{~Hz}, 1 \mathrm{H}), 2.39$ (s, $3 \mathrm{H}), 2.06-1.97$ (m, $1 \mathrm{H}), 1.92-1.82(\mathrm{~m}, 1 \mathrm{H}), 1.82-1.74(\mathrm{~m}, 1 \mathrm{H}), 1.70-1.58$ (m, $2 \mathrm{H}), 1.44$ $1.39(\mathrm{~m}, 1 \mathrm{H}), 1.05(\mathrm{~s}, 1 \mathrm{H}) ;{ }^{2} \mathrm{H}$ NMR $\left(77 \mathrm{MHz}, \mathrm{CHCl}_{3}\right) \delta 1.87$ (s, $\left.1 \mathrm{D}\right), 1.18$ (s, $\left.1 \mathrm{D}\right) ;{ }^{13} \mathrm{C}$ NMR $\left(100 \mathrm{MHz}, \mathrm{CDCl}_{3}\right) \delta 151.5,148.0,147.9,144.5,143.5,140.0,137.8,136.7,130.0,129.2,128.8$ $128.4,127.5,126.3,125.4,124.5,56.6,39.3\left(\mathrm{t}, J_{\mathrm{CD}}=18.3 \mathrm{~Hz}\right), 38.1,34.4,30.5,27.5\left(\mathrm{~d}, J_{\mathrm{CD}}=\right.$ 17.6 Hz), 21.6; IR (film) 1259, 1131, $1090 \mathrm{~cm}^{-1}$. MS (ESI) $\mathrm{m} / z 562.0739$ (562.0725 calcd for $\mathrm{C}_{25} \mathrm{H}_{23} \mathrm{D}_{2} \mathrm{~N}_{3} \mathrm{O}_{2} \mathrm{PdS}, \mathrm{M}+\mathrm{Na}^{+}$. 


\section{Selected NMR Spectra}

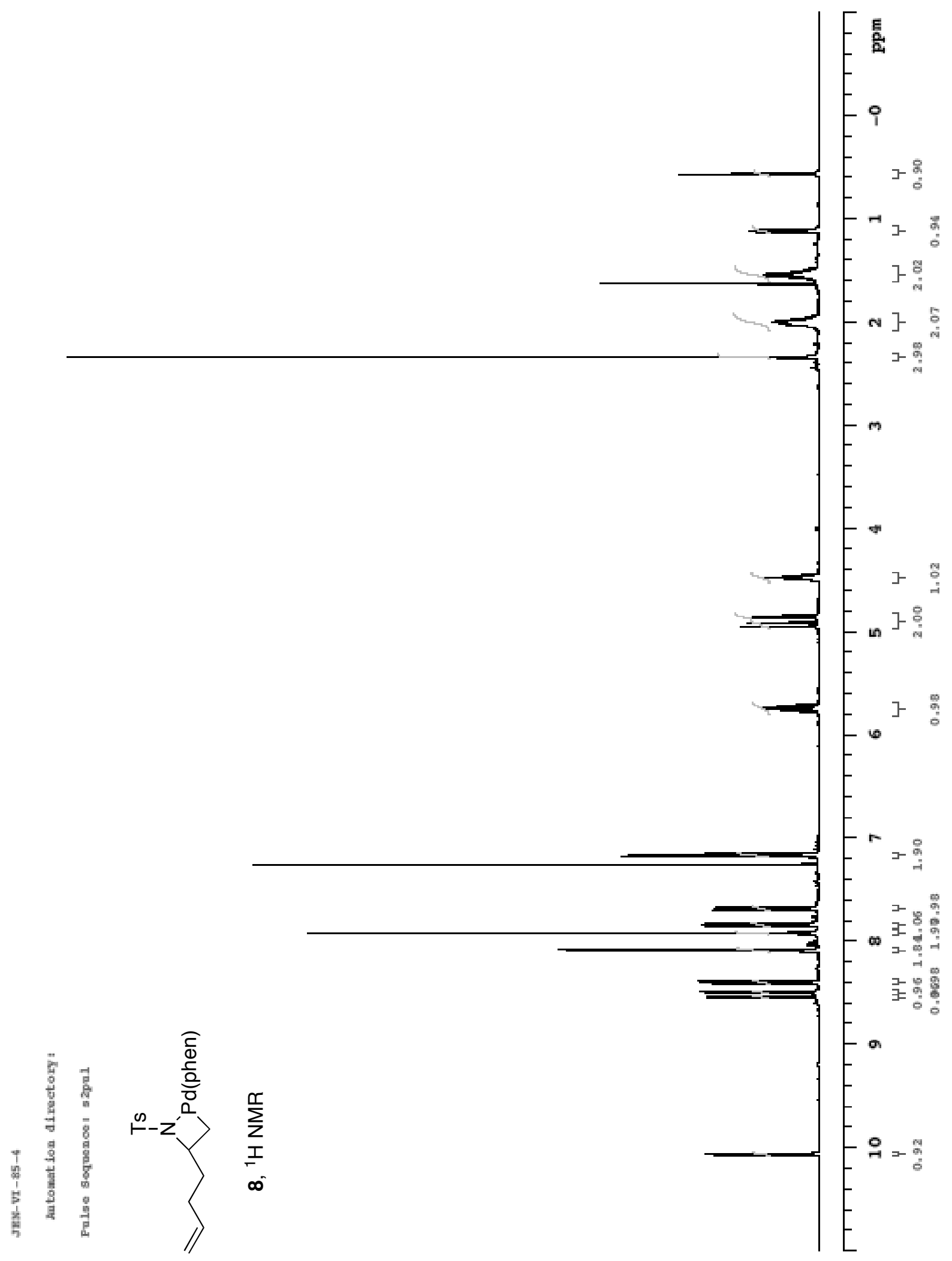


S 17

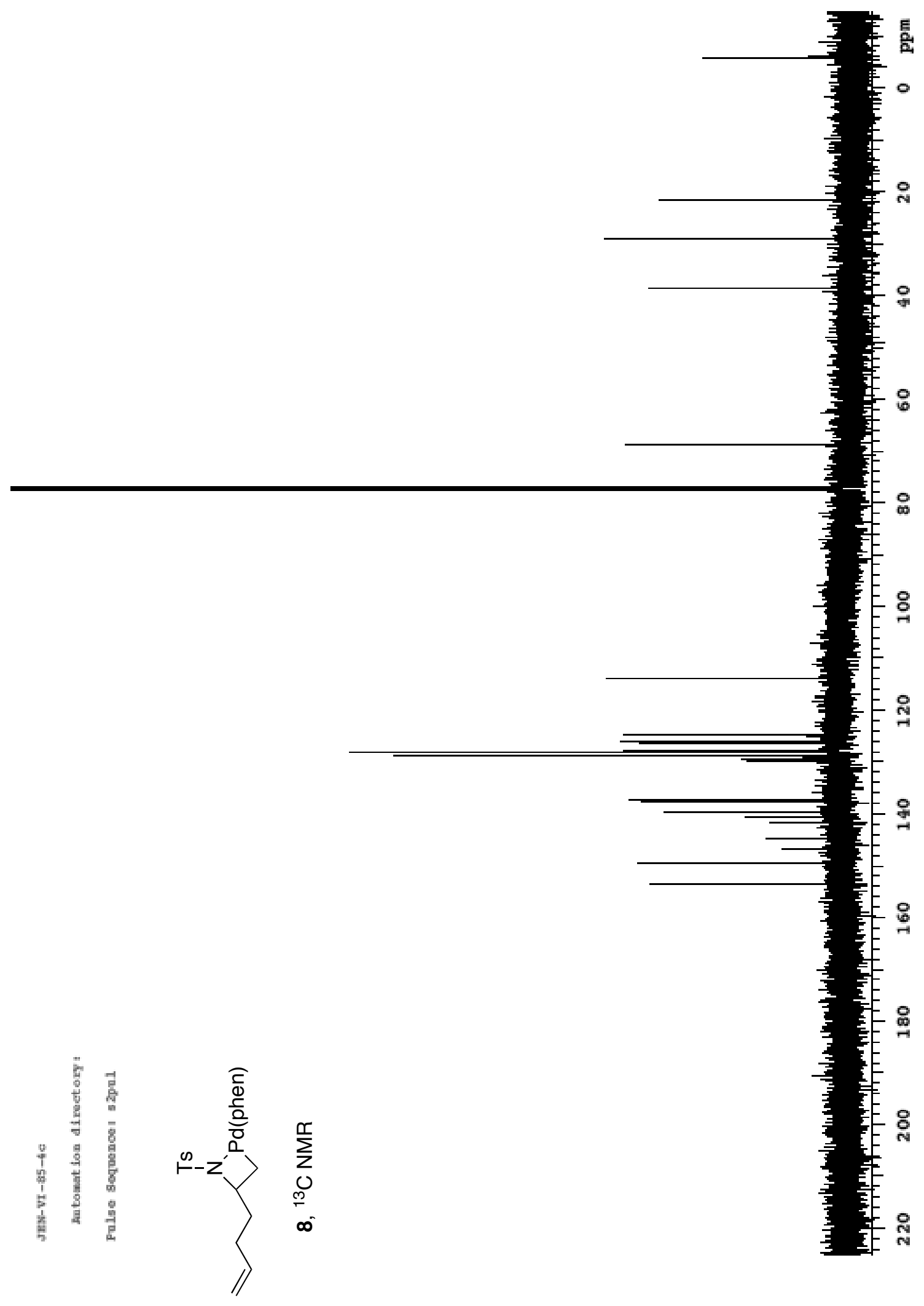




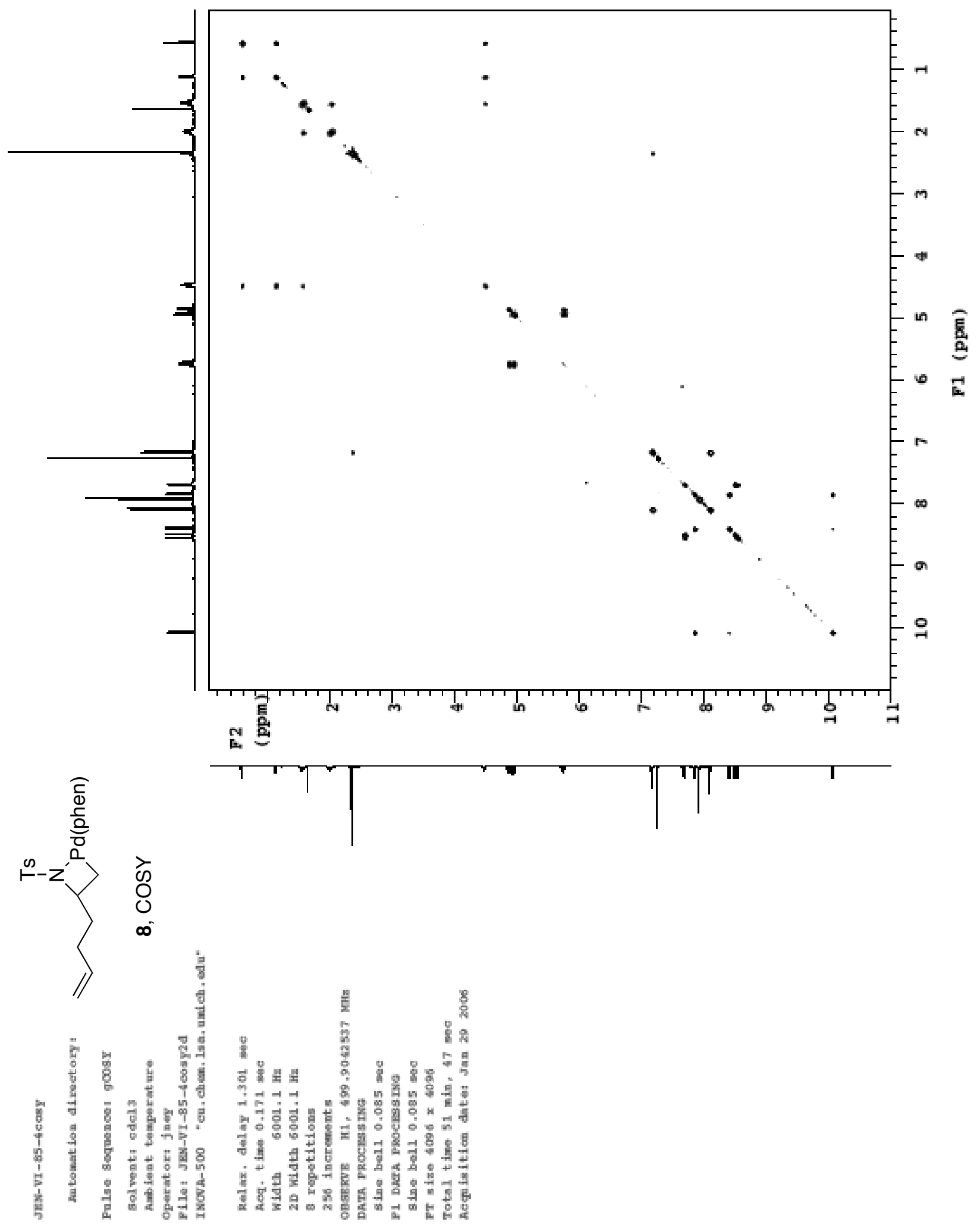




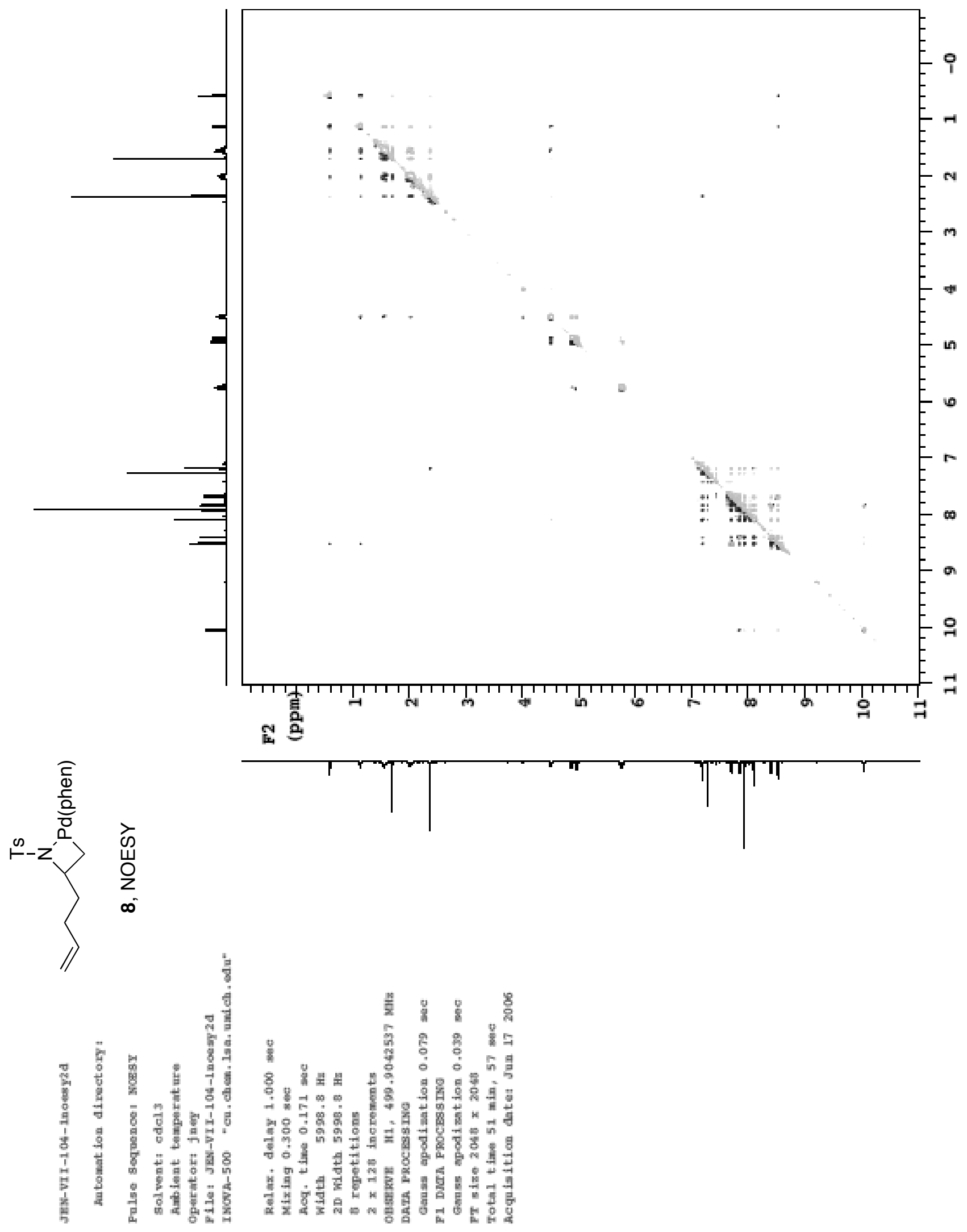




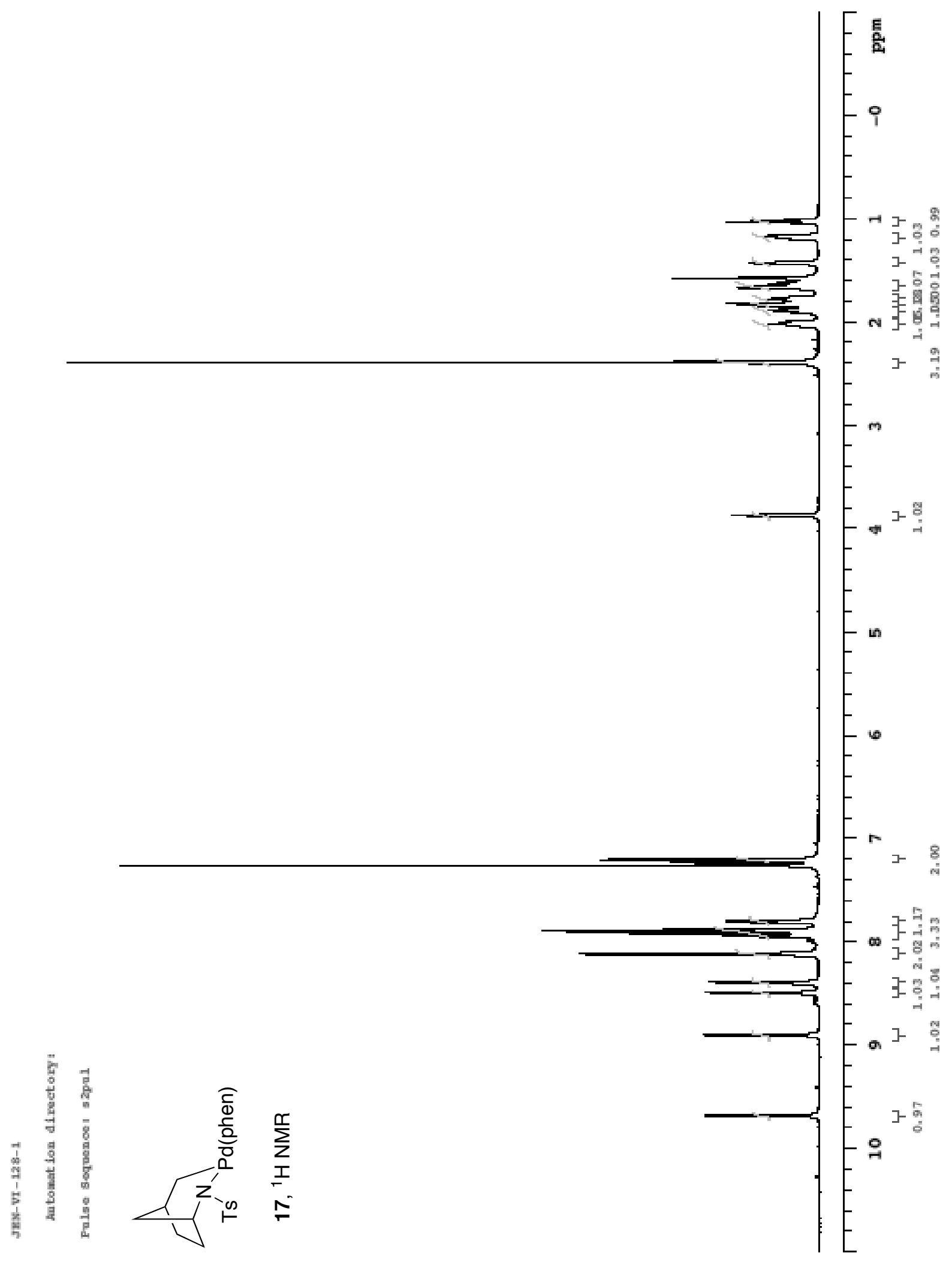


S 21

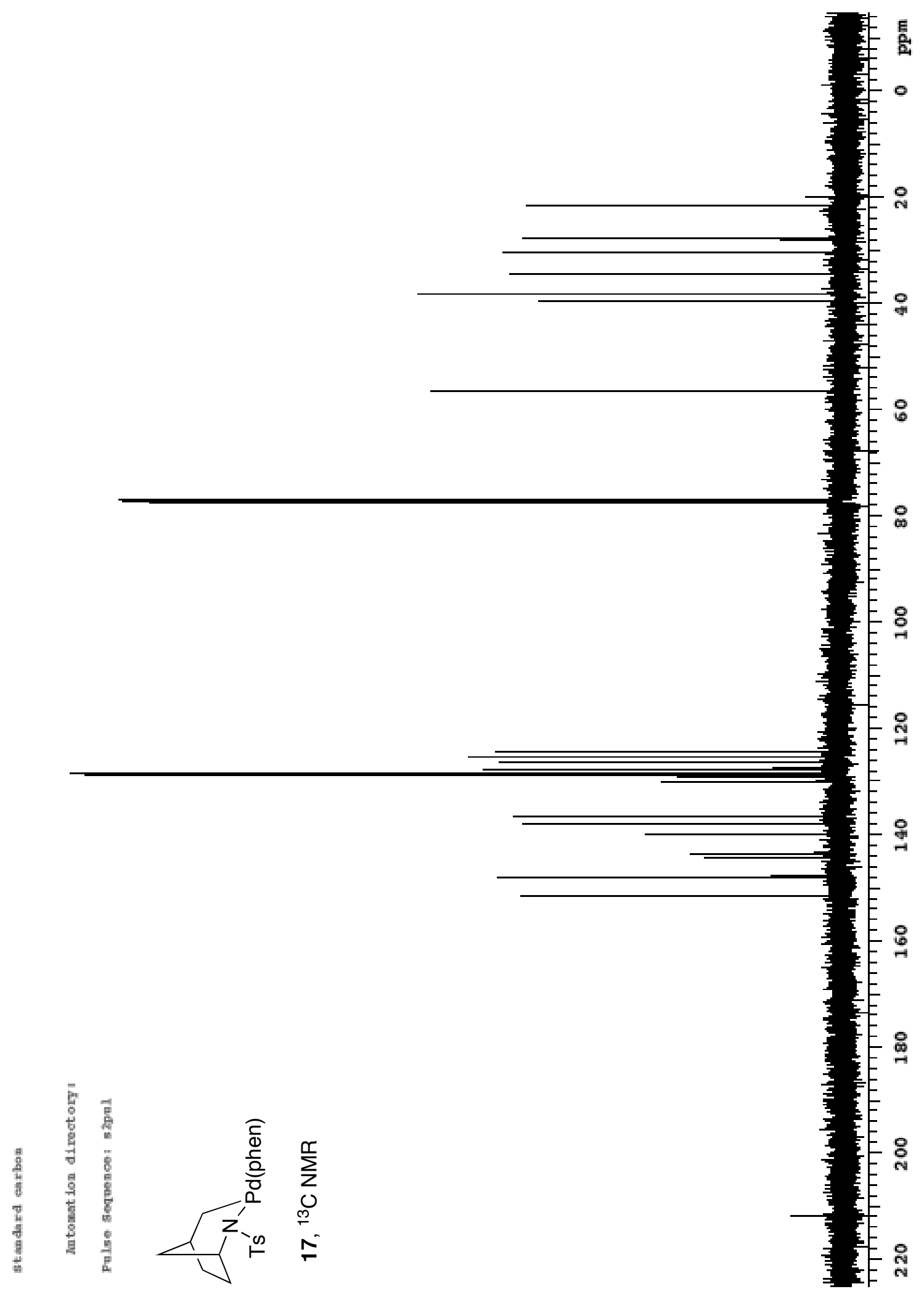




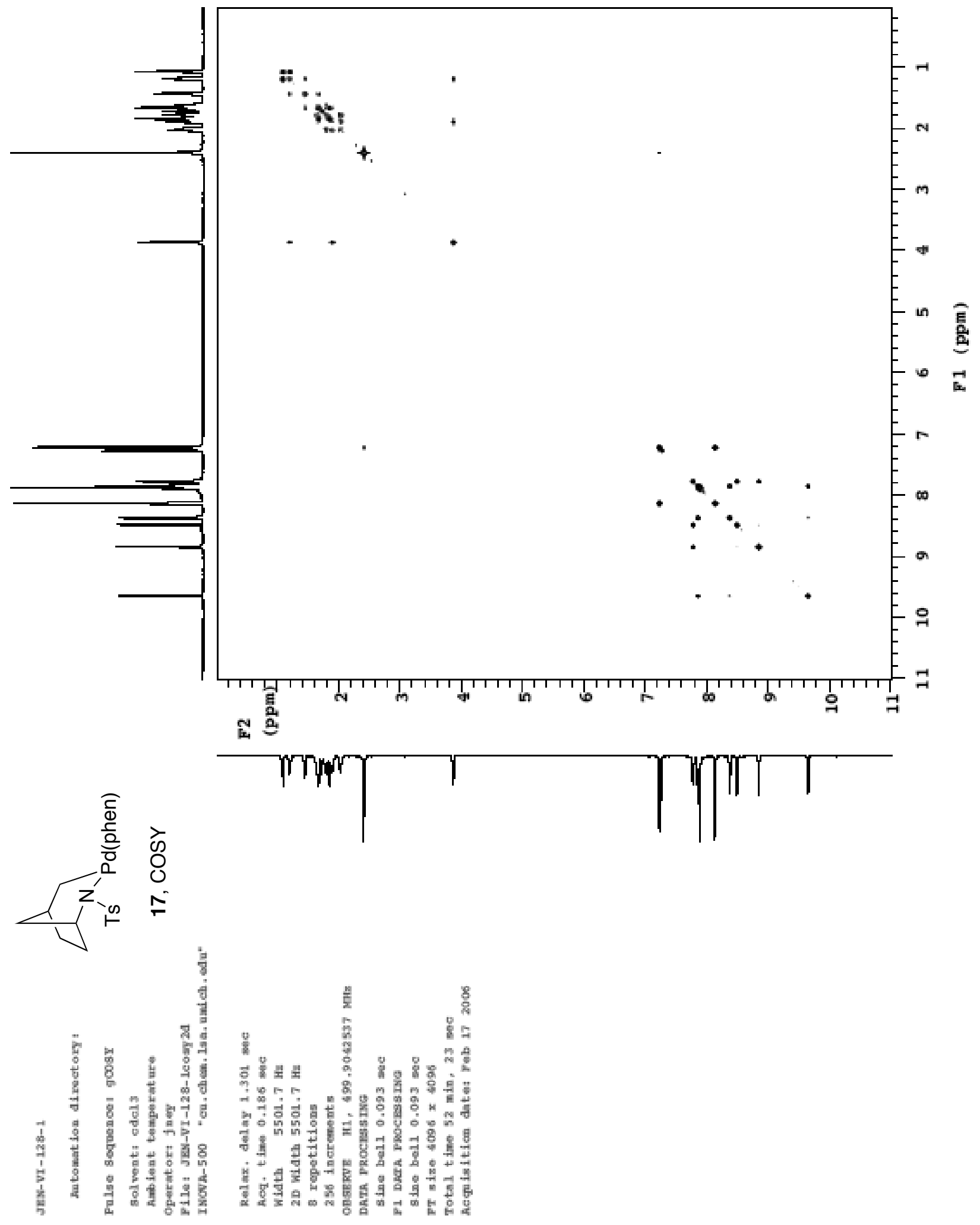




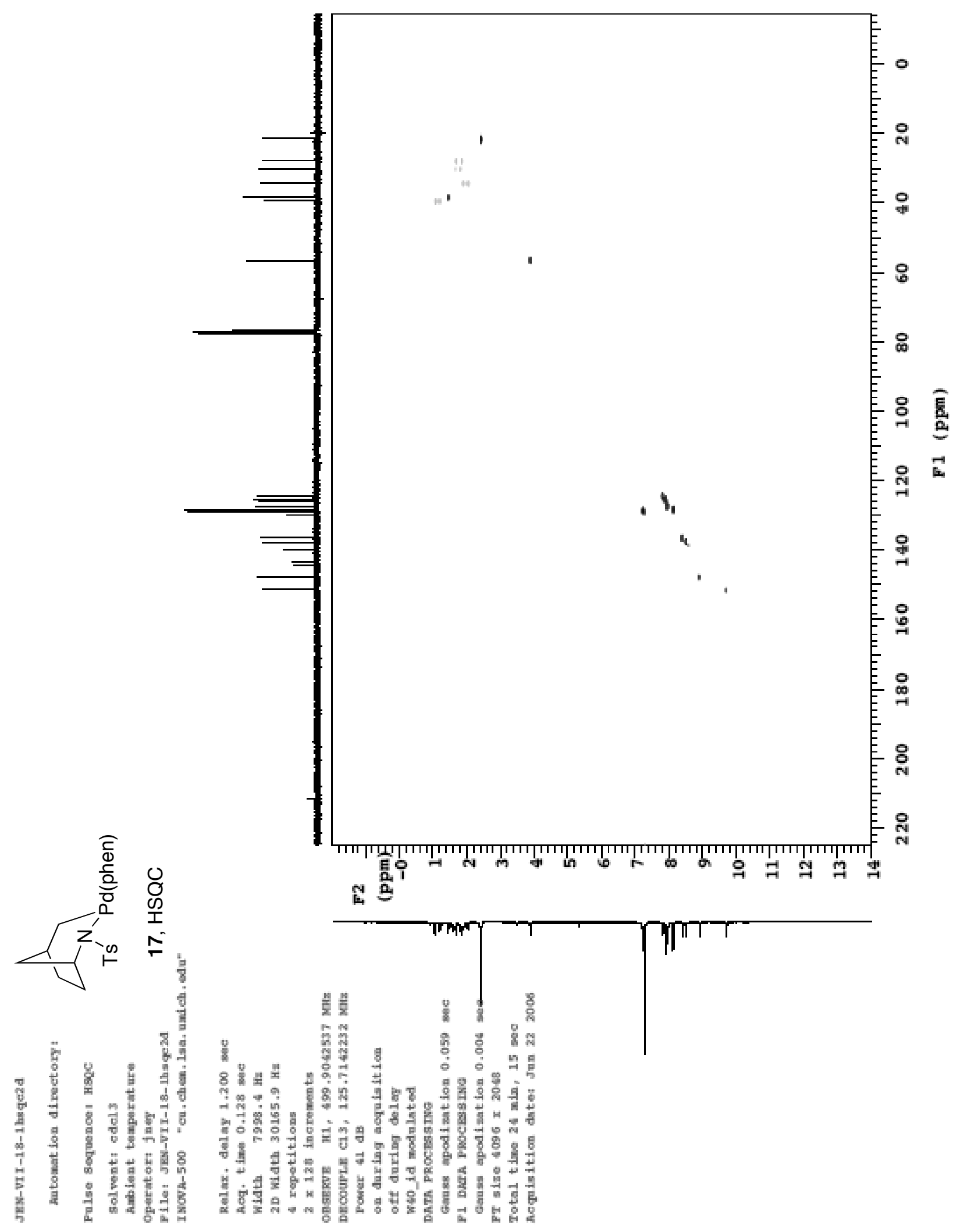




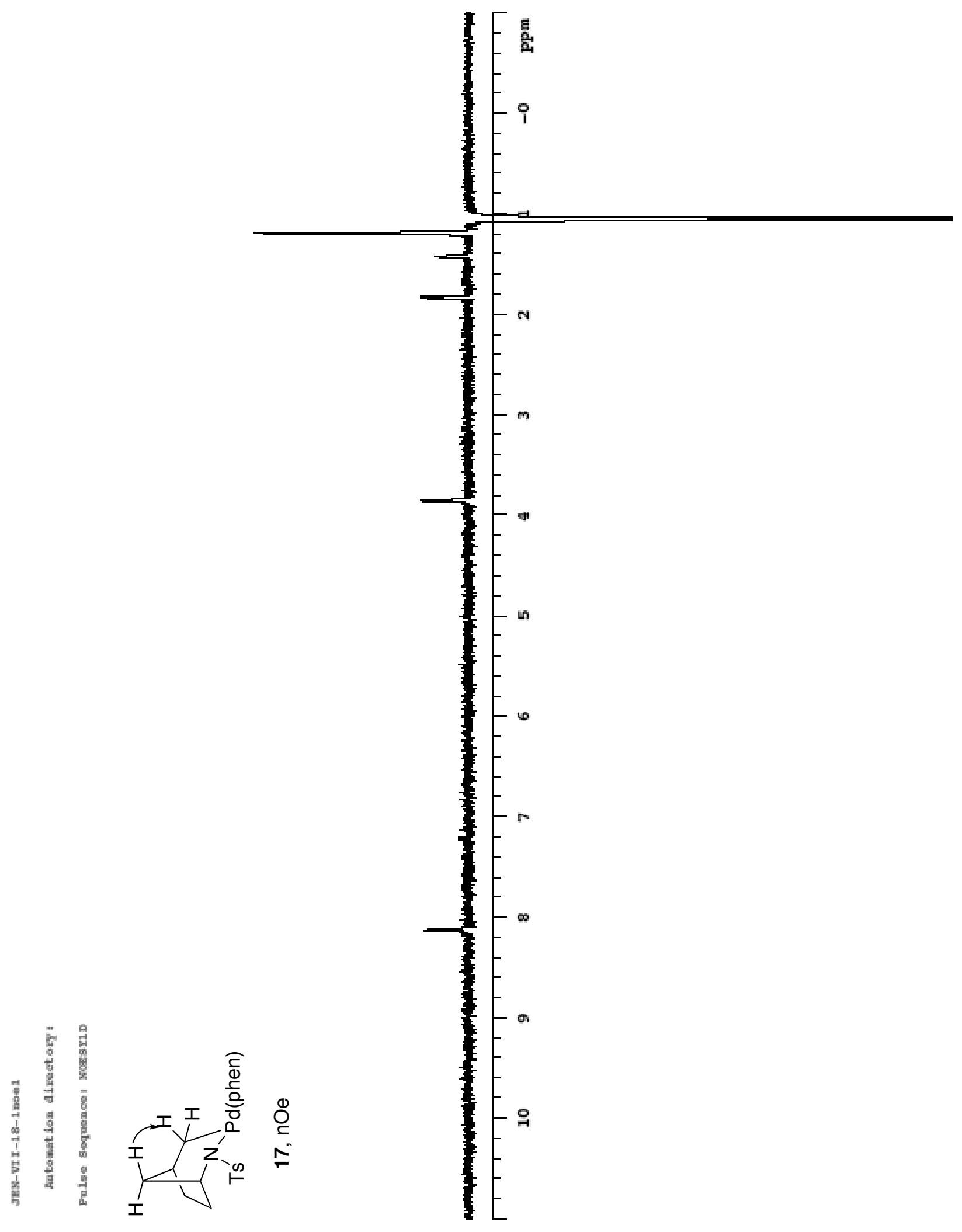




$$
\text { t }
$$




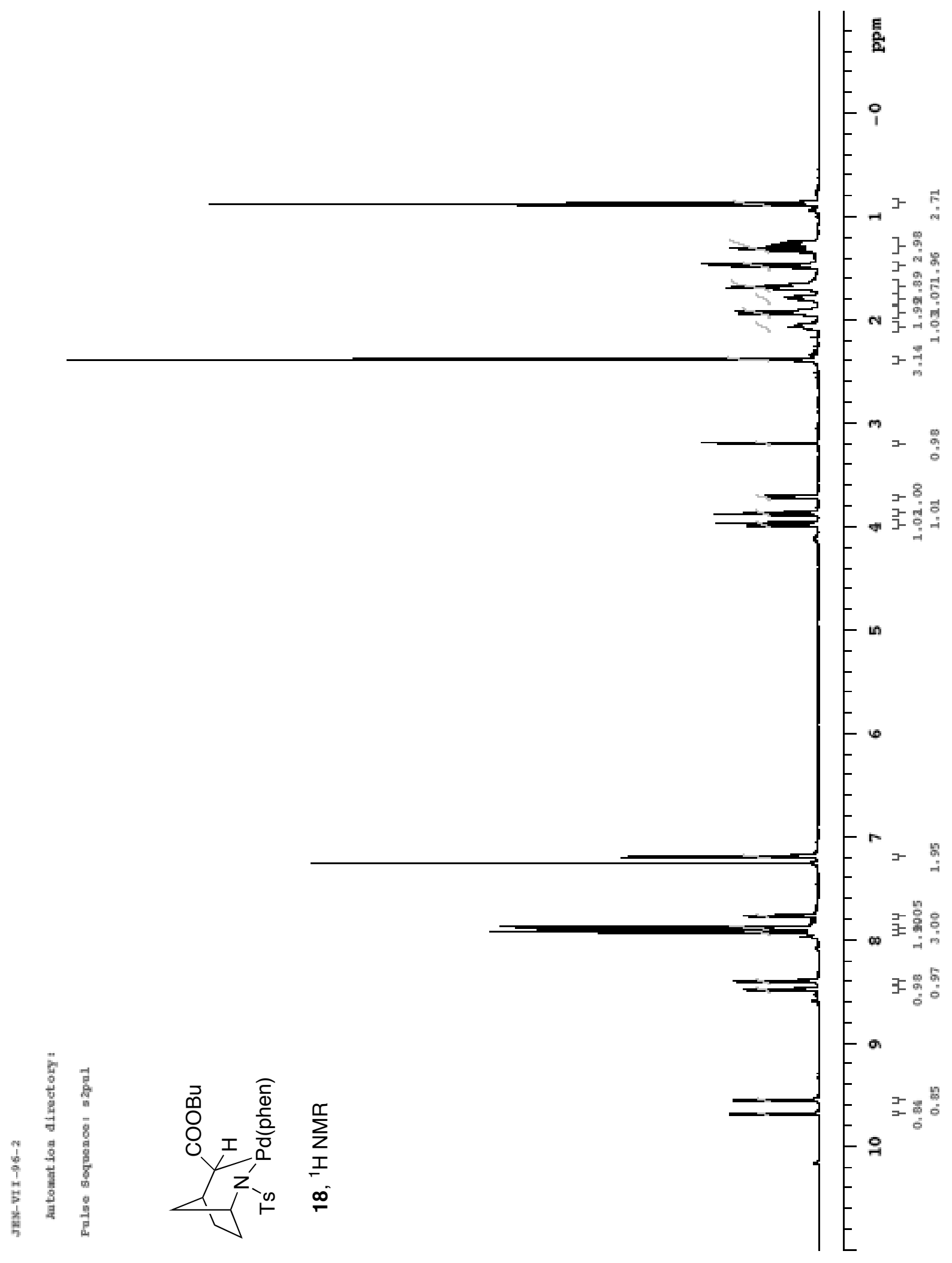




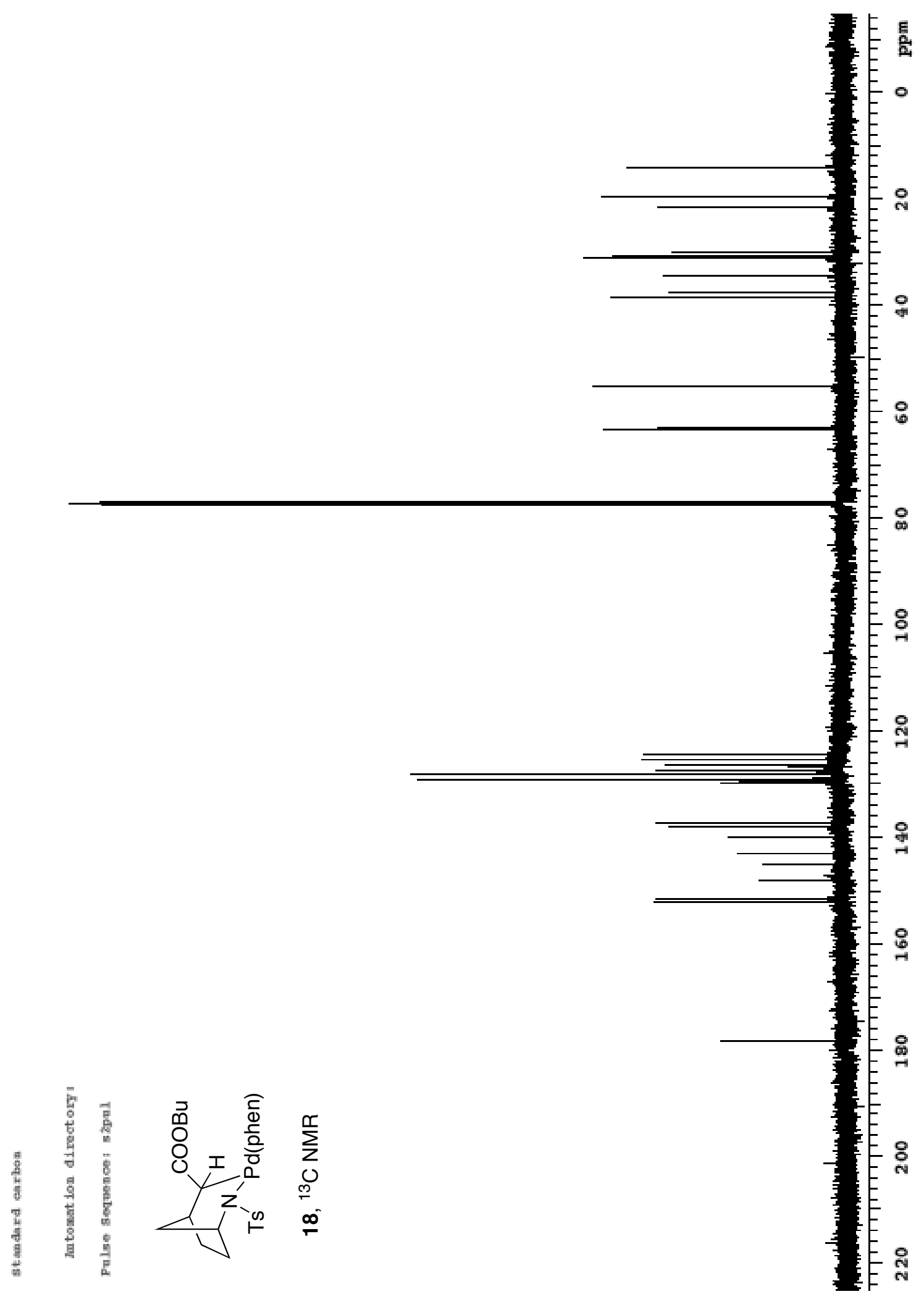




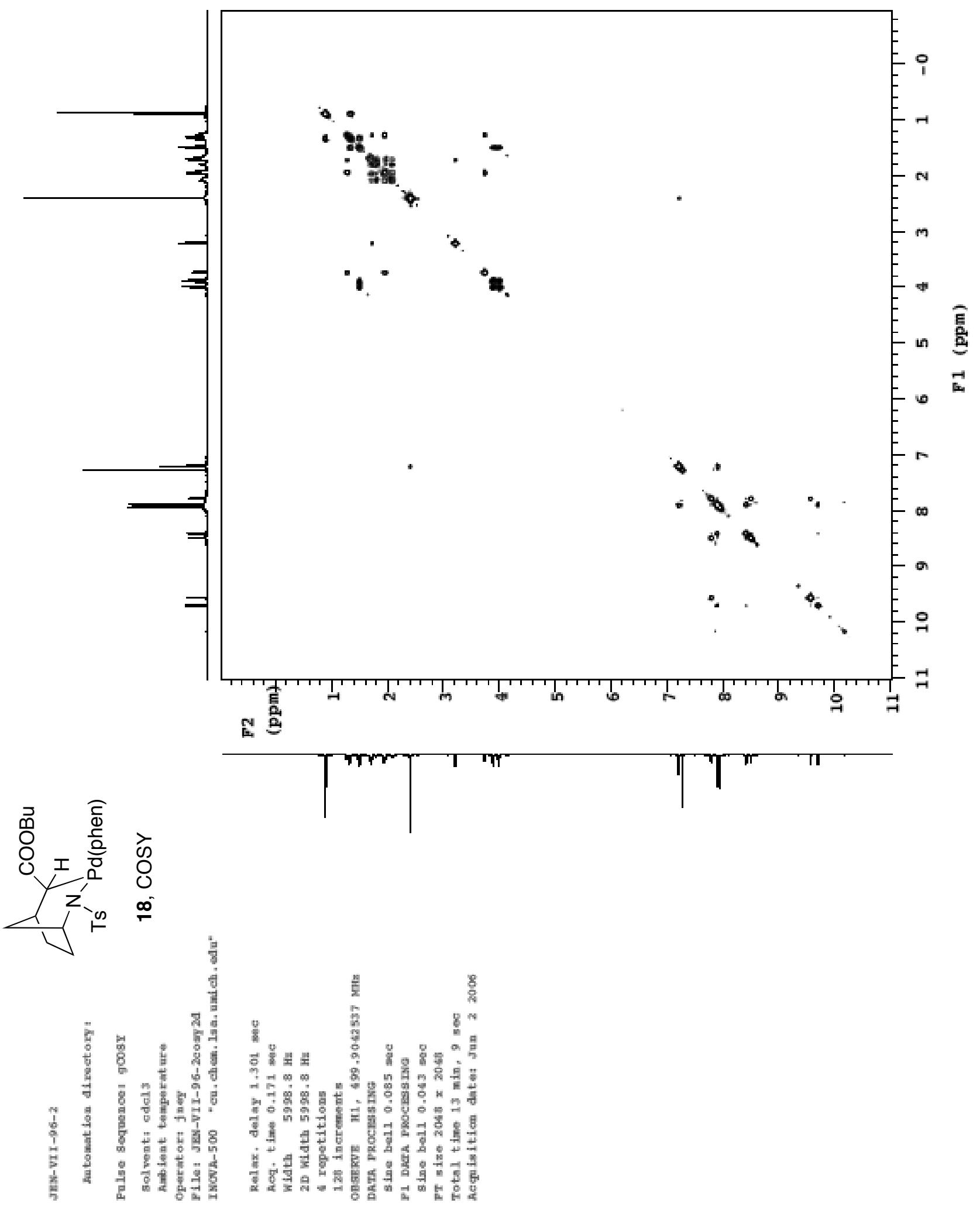




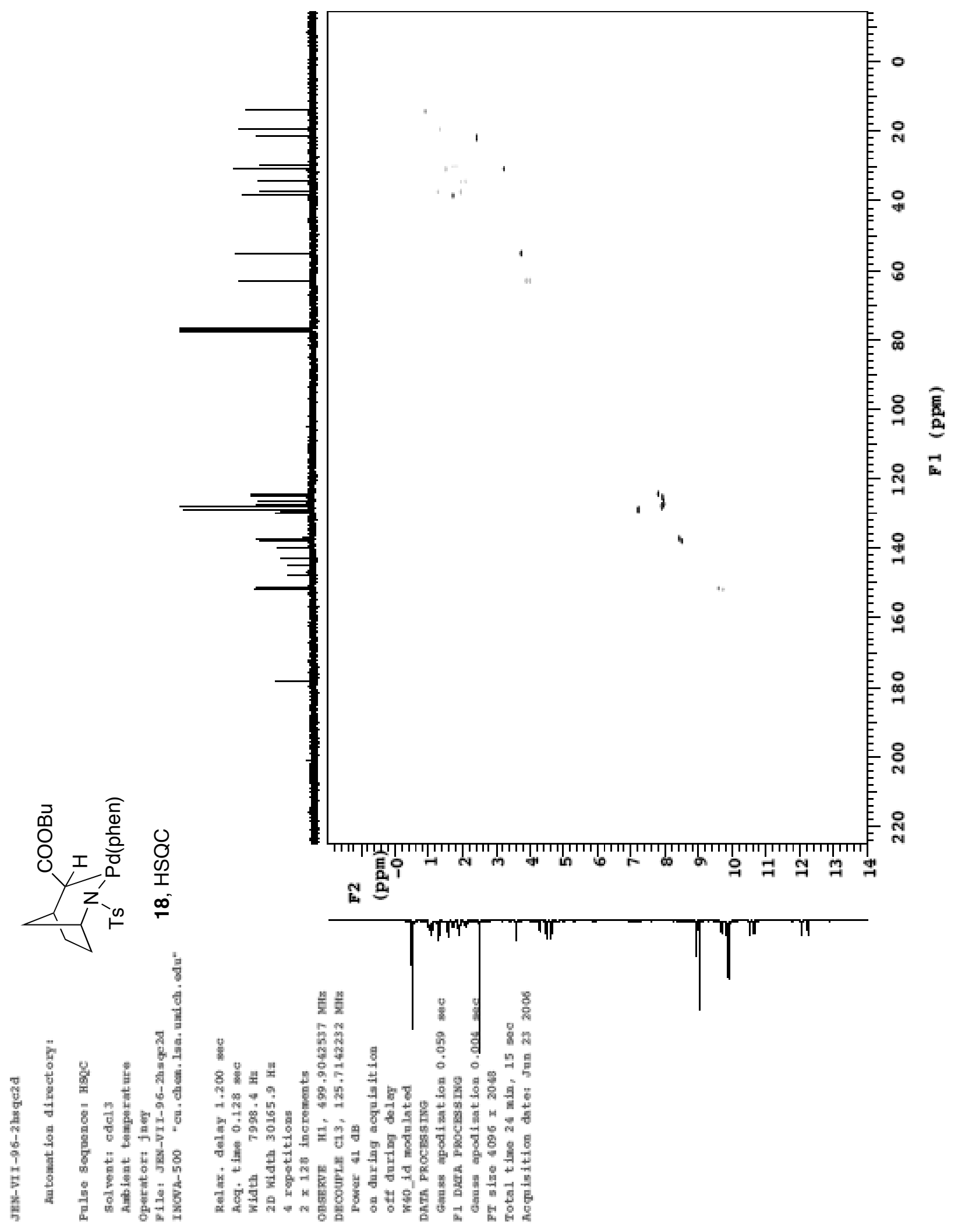




$$
\mid
$$




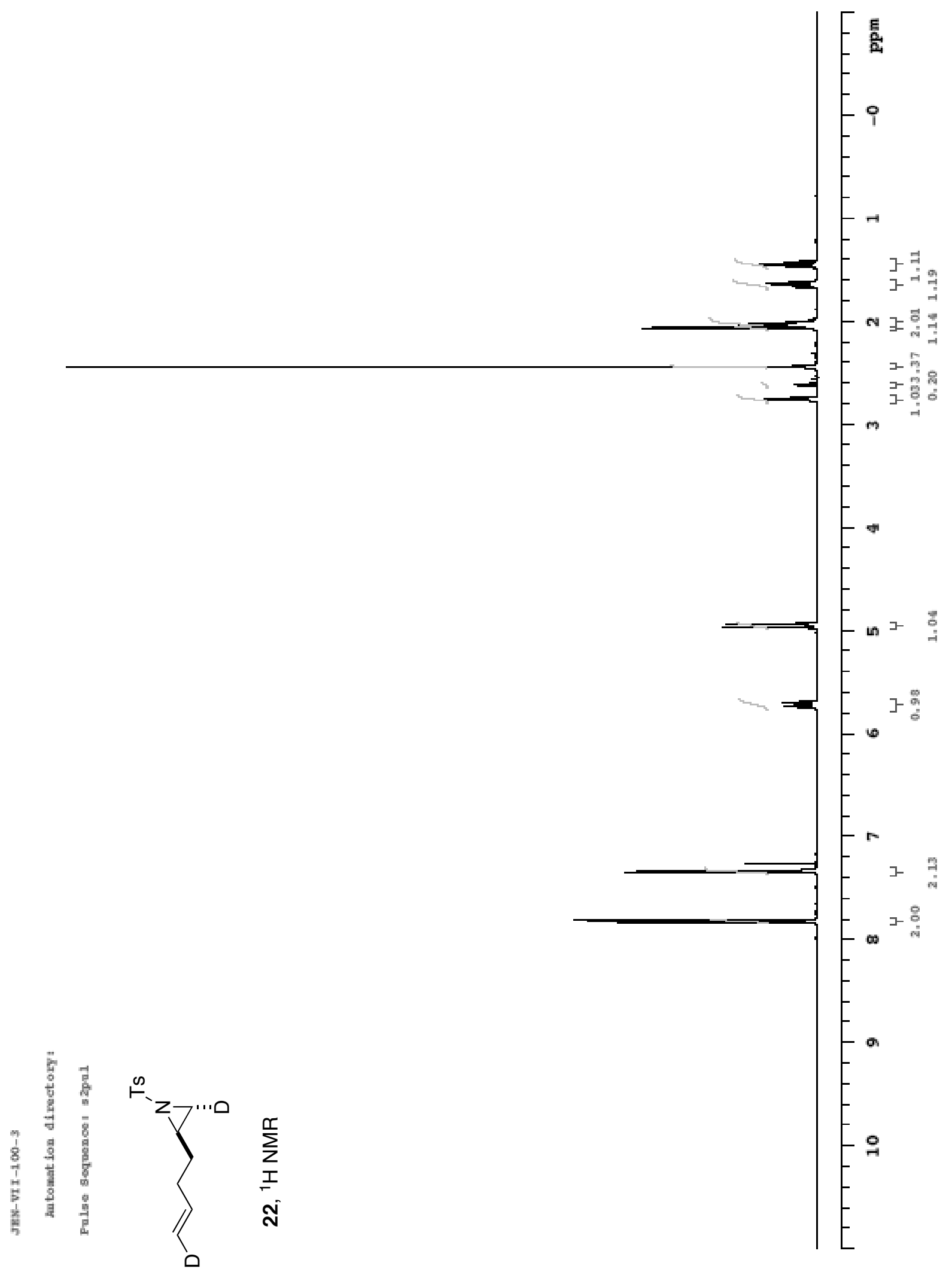




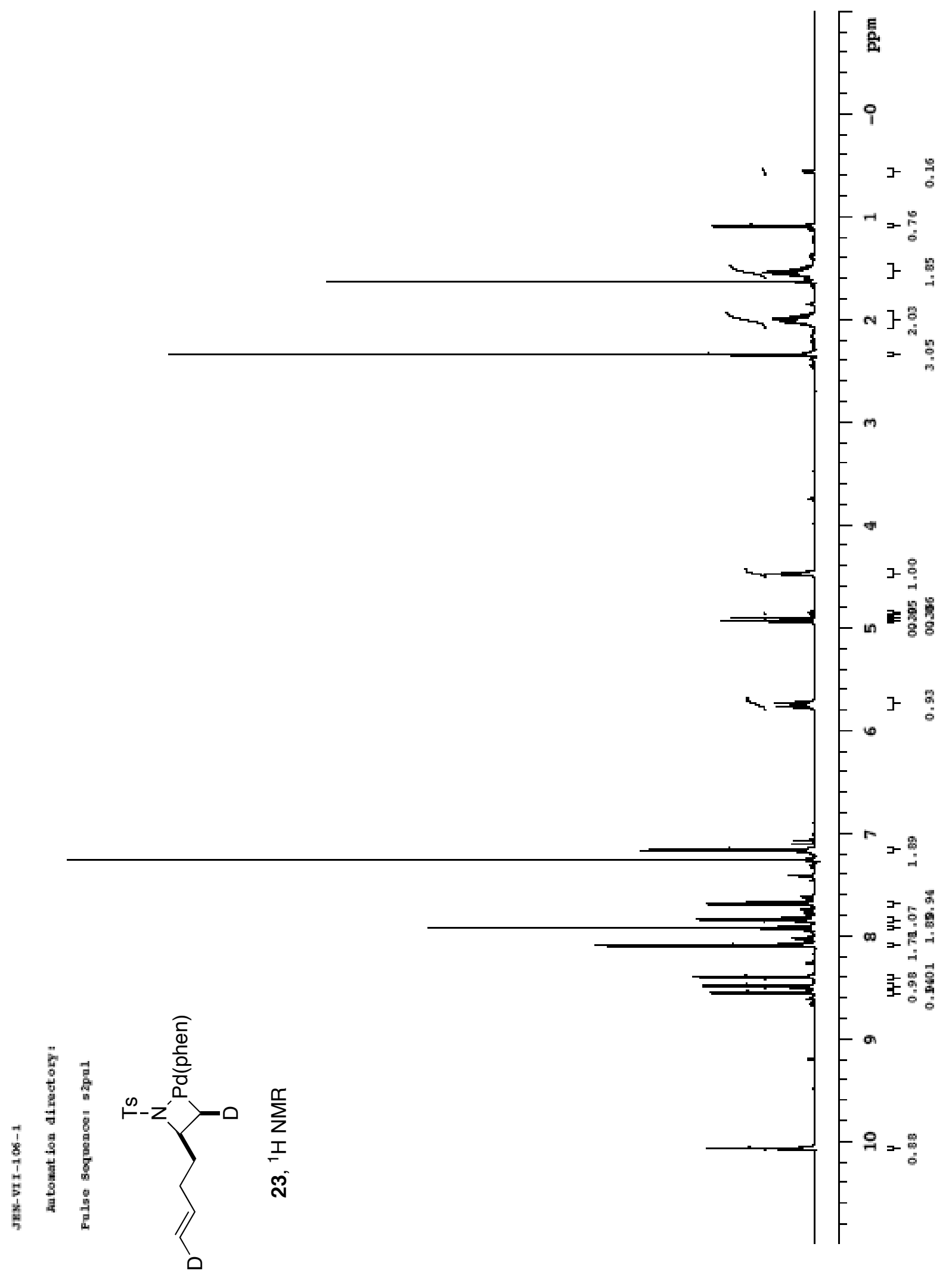




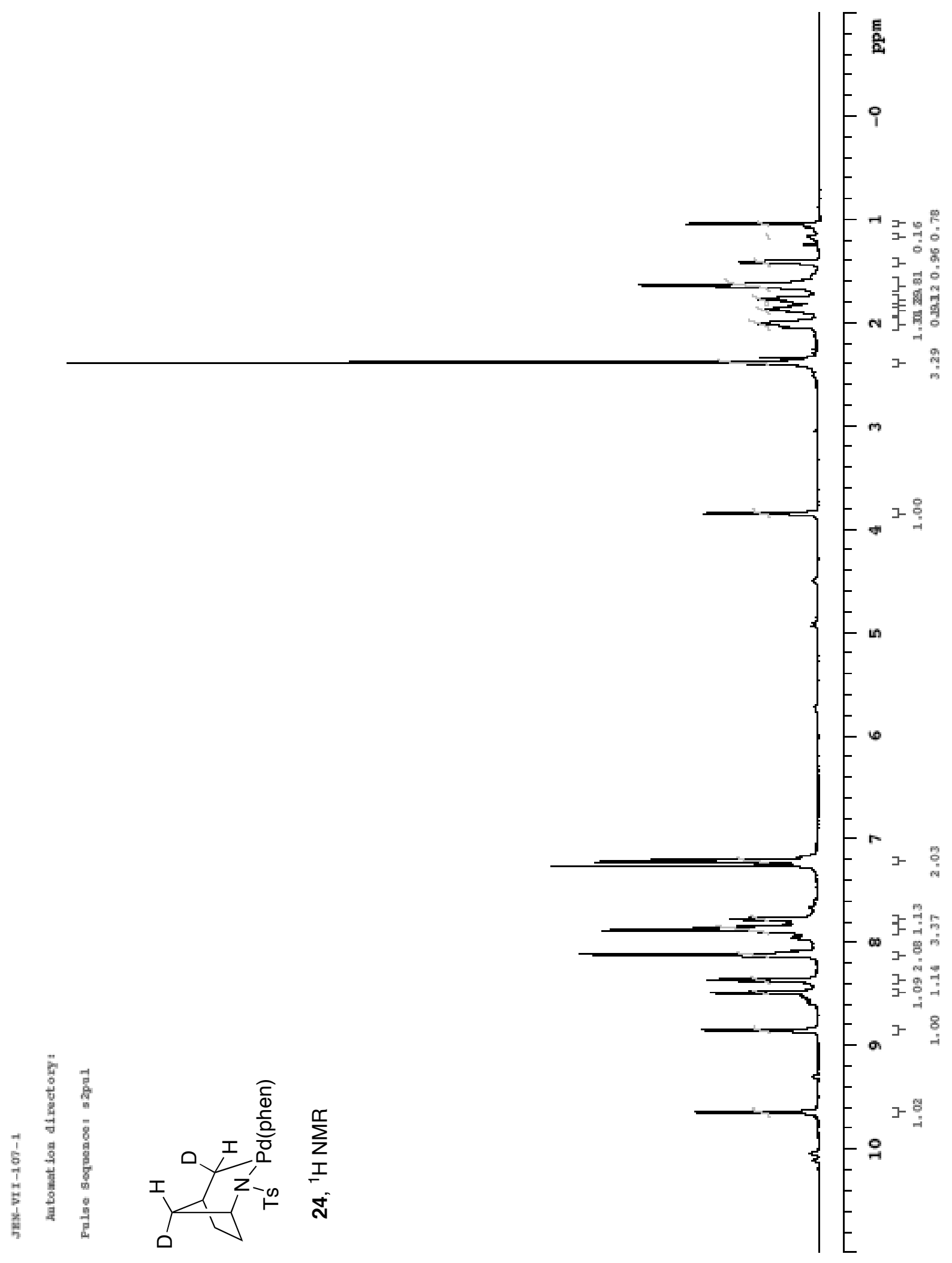




\section{References}

${ }^{1}$ Yamada, Y.; Yamamoto, T.; Okawara, M. Chem. Lett. 1975, 361-362.

${ }^{2}$ Evans, D. A.; Bilodeau, M. T.; Faul, M. M. J. Am. Chem. Soc. 1994, 116, 2742-2753.

${ }^{3}$ Nadir, U. K.; Sharma, R. L.; Koul, V. K. J. Chem. Soc., Perkin Trans. 1 1991, 2015-2019.

${ }^{4}$ Maligres, P. E.; See, M. M.; Askin, D.; Reider, P. J. Tetrahedron Lett. 1997, 38, 5253-5256.

${ }^{5}$ Han, K. I.; Pitrowski, A. M.; Eisch, J. J. in Organometallic Syntheses, Vol. 3; King, R. B.; Eisch, J. J., Eds.; Elsevier: Amsterdam, 1986; p 112.

${ }^{6}$ Piers, W. E.; Bercaw, J. E. J. Am. Chem. Soc. 1990, 112, 9406-9407.

${ }^{7}$ Wolfe, J. P.; Ney, J. E. Org. Lett. 2003, 5, 4607-4610.

${ }^{8}$ Culkin, D. A.; Hartwig, J. F. J. Am. Chem. Soc. 2001, 123, 5816-5817 and references cited therein. 